\title{
The Distribution of Second Degrees in the Buckley-Osthus Random Graph Model
}

\author{
Andrey Kupavskii, Liudmila Ostroumova, Dmitriy Shabanov, \\ and Prasad Tetali
}

Abstract. In this article we consider a well-known generalization of the Barabási and Albert preferential attachment model - the Buckley-Osthus model. Buckley and Osthus proved that in this model, the degree sequence has a power law distribution. As a natural (and arguably more interesting) next step, we study the second degrees of vertices. Roughly speaking, the second degree of a vertex is the number of vertices at distance two from the given vertex. The distribution of second degrees is of interest because it is a good approximation of PageRank, where the importance of a vertex is measured by taking into account the popularity of its neighbors.

We prove that the second degrees also obey a power law. More precisely, we estimate the expectation of the number of vertices with the second degree greater than or equal to $k$ and prove the concentration of this random variable around its expectation using the now-famous Talagrand's concentration inequality over product spaces. As far as we know, this is the only application of Talagrand's inequality to random web graphs where the (preferential attachment) edges are not defined over a product distribution, making the application nontrivial and requiring a certain degree of novelty. 


\section{Introduction}

In this paper we consider some properties of random graphs. The standard random graph model $G(n, m)$ was introduced in [Erdős and Rényi 59]. In this model, we randomly choose one graph from all graphs with $n$ vertices and $m$ edges. A similar model $G(n, p)$ was suggested in [Gilbert 59]: we have an $n$-vertex set, and we join each pair of vertices independently with probability $0<p<1$. Many papers deal with the classical models. Fundamental results about these models can be found in [Bollobás 01, Erdős and Rényi 60, Janson et al. 00].

Recently, there has been increased interest in modeling complex real-world networks. It is well understood that real structures differ from standard random graphs. Many models of real-world networks and main results can be found in [Bollobás and Riordan 03]. For example, a basic characteristic of random graphs is their degree sequence. In many real-world structures, the degree sequence obeys a power law distribution. However, standard random graph models do not have this property.

In [Barabási and Albert 99], the so-called preferential attachment model was proposed, which has the desired degree distribution. Later, a more precise definition of this model was given in [Bollobás et al. 01]. In this model, the probability that a new vertex is connected to some previous vertex $v$ is proportional to the degree of $v$. Bollobás and Riordan also proved that the degree sequence has a power law distribution with exponent equal to -3 .

Naturally, one would not expect that this constant would suit all (or even most) real networks. In order to make the model more flexible, two groups of authors (see [Dorogovtsev et al. 00] and [Drinea et al. 01]) have proposed adding one more parameter - the "initial attractiveness" of a node, which is a positive constant that does not depend on the degree. An explicit construction of that model is given in [Buckley and Osthus 04].

Many papers deal with variations of preferential attachment. We mention here the paper [Rudas et al. 07], the authors of which consider quite a generic model of a random tree and prove some interesting results concerning the neighborhood structure of a random vertex. Also, one can find a neighborhood analysis in preferential attachment models in the preprint [Berger et al. 09] on the weak graph limit.

This paper deals with the Buckley-Osthus model, which we now describe. Let $n$ be the number of vertices in our graph, and let $m \in \mathbb{N}$ and $a \in \mathbb{R}_{+}$be fixed parameters.

We begin with the case $m=1$. We inductively construct a random graph $H_{a, 1}^{n}$. The graph $H_{a, 1}^{1}$ consists of one vertex and one loop (we can also start with $H_{a, 1}^{0}$, which is the empty graph). Assume that we have already constructed the graph 
$H_{a, 1}^{t-1}$. At the next step, we add one vertex $t$ and one edge between vertices $t$ and $i$, where $i$ is chosen randomly with

$$
\mathrm{P}(i=s)= \begin{cases}\frac{d_{H_{a, 1}^{t-1}}(s)-1+a}{(a+1) t-1} & \text { if } 1 \leq s \leq t-1, \\ \frac{a}{(a+1) t-1} & \text { if } s=t\end{cases}
$$

Here $d_{H_{a, 1}^{t}}(s)$ is the degree of the vertex $s$ in $H_{a, 1}^{t}$. We will also use the notation $d(s):=d_{H_{a, 1}^{n}}(s)$.

To construct $H_{a, m}^{n}$ with $m>1$, we start from $H_{a, 1}^{m n}$. Then we identify the vertices $1, \ldots, m$ to form the first vertex; we identify the vertices $m+1, \ldots, 2 m$ to form the second vertex, etc. As for the edges, if the edge $e$ connects vertices $i m+k$ and $j m+l, 1 \leq k, l \leq m$, in the graph $H_{a, 1}^{m n}$, then we draw an edge $e^{\prime}$ between vertices $i+1$ and $j+1$ in $H_{a, m}^{n}$. Note that we have a one-to-one correspondence between the edges of $H_{a, 1}^{m n}$ and $H_{a, m}^{n}$, so there may be multiple edges (and multiple loops) between vertices in $H_{a, m}^{n}$. Denote by $\mathfrak{H}_{a, m}^{n}$ the probability space of constructed graphs. In this paper we mainly study the case $m=1$.

Here we mainly focus on the case of undirected graphs $H_{a, m}^{n}$. However, each edge has a natural orientation, from a later vertex to an earlier one. In some cases, it is convenient to deal with oriented edges, so sometimes we use the term outgoing edge.

In [Buckley and Osthus 04], it is proved that the degree sequence of $H_{a, m}^{n}$ has a power law with exponent $-2-a$, where $a$ is a natural number. Recently, Grechnikov substantially improved this result.

Theorem I.I. [Grechnikov 13] Let $a \in \mathbb{R}_{+}$. If $d=d(n) \geq m$ and $\psi(n) \rightarrow \infty$ as $n \rightarrow \infty$, then

$$
\left|R(d, n)-\frac{\mathrm{B}(d-m+m a, a+2) n}{\mathrm{~B}(m a, a+1)}\right| \leq\left(\sqrt{d^{-2-a} n}+d^{-1}\right) \psi(n),
$$

with probability tending to 1 as $n \rightarrow \infty$. Here $R(d, n)$ is the number of vertices in $H_{a, m}^{n}$ with degree equal to $d$, and $\mathrm{B}(x, y)$ is the beta function.

The edge distribution (i.e., the number of edges between vertices of given degrees) of the Buckley-Osthus model was considered in [Grechnikov 13]. Based on this result, the authors of [Zhukovskiy et al. 12] showed that both the degree distribution and the edge distribution of the web host graph can be modeled by the Buckley-Osthus model. Therefore, it is reasonable to analyze other properties of this model and compare them to properties of real-world networks. 
In this paper we consider the so-called second degrees of vertices. Roughly speaking, the second degree of a vertex is the number of vertices at distance two from the given vertex. The distribution of second degrees is of interest because it is a good approximation of PageRank [Page et al. 98], where, informally speaking, the importance of a vertex depends on the importance (degree, popularity) of its neighbors.

We prove that the number of vertices $Y_{n}(k)$ with second degree at least $k$ decreases as $k^{-a}$, where $a$ is the initial attractiveness. This means that the distribution of second degrees obeys a power law. To prove this, we calculate the expectation of $Y_{n}(k)$ and show the concentration of this random variable around its expectation using Talagrand's inequality. The application of this inequality is nontrivial. In particular, we have to redefine the probability space of the Buckley-Osthus graph so that we obtain a product probability space with a product measure. After that, we use Talagrand's inequality in its general asymmetric form, which is essential. Verifying the hypothesis of Talagrand's theorem for the present purpose turns out to be delicate, requiring us to introduce additional combinatorial constructions.

It would be interesting to study the distribution of second degrees in real-world networks. If this distribution follows a power law with the predicted exponent, then this is another advantage of preferential attachment models. We leave experimental analysis of real-world networks for future work.

This paper is organized as follows. In Section 2, we give the main definitions and formulate the results. In Sections 3 and 4, we prove the theorems stated in Section 2.

\section{Definitions and Results}

\section{I. Definitions}

In this paper we study the random graph $H_{a, 1}^{n}$. We shall write $i j \in H_{a, 1}^{n}$ if $H_{a, 1}^{n}$ contains the edge $i j$; we shall write $t \in H_{a, 1}^{n}$ if $t$ is a vertex of $H_{a, 1}^{n}$. Given a vertex $t \in H_{a, 1}^{n}$, the second degree of the vertex $t$ is

$$
d_{2}(t)=\#\left\{i j: i \neq t, j \neq t, i t \in H_{a, 1}^{n}, i j \in H_{a, 1}^{n}\right\} .
$$

In other words, the second degree of $t$ is the number of edges adjacent to the neighbors of $t$ except for the edges adjacent to the vertex $t$. We say that a vertex $t$ is a $k$-vertex if $d_{2}(t) \geq k$.

Let $M_{n}^{1}(d)$ be the expectation of the number of vertices with degree $d$ in $H_{a, 1}^{n}$ :

$$
M_{n}^{1}(d)=\mathrm{E}\left(\#\left\{t \in H_{a, 1}^{n}: d_{H_{a, 1}^{n}}(t)=d\right\}\right) .
$$


Let $Y_{n}(k)$ denote the number of $k$-vertices in $H_{a, 1}^{n}$.

In this paper we study second degrees of vertices in $H_{a, 1}^{n}$. The main results are stated in Theorems 2.1 and 2.8.

We also consider the variable $X_{n}(k)$ equal to the number of vertices with second degree $k$ in $H_{a, 1}^{n}$. Note that $Y_{n}(k)=\sum_{i \geq k} X_{n}(i)$.

\subsection{Expectation}

Theorem 2.I. For every $k>1$, we have

$$
\mathrm{E} Y_{n}(k)=\frac{(a+1) \Gamma(2 a+1)}{\Gamma(a+1) k^{a}} n\left(1+O\left(\frac{(\ln k)^{\lceil a+1\rceil}}{k}\right)+O\left(\frac{k^{1+a}}{n}\right)\right)
$$

An easy consequence of Theorem 2.1 is the following corollary.

Corollary 2.2. We have $\mathrm{E} Y_{n}(k)=\Theta\left(\frac{n}{k^{a}}\right)$ for $k=O\left(n^{\frac{1}{1+a}}\right)$.

Using the same technique as in proof of Theorem 2.1 we can prove the following result.

Theorem 2.3. For every $k \geq 1$, we have

$$
\mathrm{E} X_{n}(k)=\frac{(a+1) \Gamma(2 a+1) n}{\Gamma(a) k^{a+1}}\left(1+O\left(\frac{(\ln k)^{\lceil a+1\rceil}}{k}\right)+O\left(\frac{k^{1+a}}{n}\right)\right) .
$$

Again, as a consequence we get the following.

Corollary 2.4. We have $\mathrm{E} X_{n}(k)=\Theta\left(\frac{n}{k^{1+a}}\right)$ for $k=O\left(n^{\frac{1}{1+a}}\right)$.

Let $N_{n}(l, k)$ be the number of vertices in $H_{a, 1}^{n}$ with degree $l$, with second degree $k$, and without loops:

$$
N_{n}(l, k)=\#\left\{t \in H_{a, 1}^{n}: d(t)=l, d_{2}(t)=k, t t \notin H_{a, 1}^{n}\right\} .
$$

To prove Theorem 2.1, we need the following auxiliary theorem.

Theorem 2.5. In $H_{a, 1}^{n}$ we have

$$
\mathrm{E} N_{n}(l, k)=c(l, k)(n+\theta(n, l, k))
$$


where $\theta(n, l, k)$ is some function on $n, l, k$ such that $|\theta(n, l, k)|<C(l+k)^{1+a}$ with an absolute constant $C$. The constants $c(l, k)$ are defined as follows:

$$
\begin{aligned}
& c(l, 0)=c(0, k)=0, \\
& c(1, k)=c(1, k-1) \frac{a+k-1}{k+3 a+1}+c(k) \frac{a+k-1}{k+3 a+1}, \quad k>0, \\
& c(l, k)=c(l, k-1) \frac{a l+k-1}{l(1+a)+k+2 a}+c(l-1, k) \frac{l-2+a}{l(1+a)+k+2 a}, \\
& k>0, l>1 . \\
& \text { Here } c(k)=\frac{\mathrm{B}(k-1+a, a+2)}{\mathrm{B}(a, a+1)} .
\end{aligned}
$$

To prove these theorems we shall use two lemmas. The following result was obtained in [Grechnikov 13].

Lemma 2.6. Let $k \geq 1$ be a natural number. Then

$$
M_{n}^{1}(k)=\frac{\mathrm{B}(k-1+a, a+2) n}{\mathrm{~B}(a, a+1)}+\tilde{\theta}(n, k),
$$

where $\tilde{\theta}(n, k)$ is some function on $n$ and $k$ such that $|\tilde{\theta}(n, k)|<\tilde{C} / k$ with an absolute constant $\tilde{C}$.

Denote by $P_{n}(l, k)$ the number of vertices in $H_{a, 1}^{n}$ with a loop, with degree $l$, and with second degree $k$.

Lemma 2.7. For every $n$, we have

$$
\mathrm{E} P_{n}(l, k) \leq p(l, k),
$$

where

$$
\begin{aligned}
p(2,0) & =P, \\
p(l, 0) & =p(l-1,0) \frac{l-2+a}{l(1+a)-2-a}, \quad l \geq 3, \\
p(l, k) & =p(l, k-1) \frac{a l+k-2 a-1}{l(1+a)+k-1-a}+p(l-1, k) \frac{l-2+a}{l(1+a)+k-1-a}, \\
l & \geq 3, k \geq 1 .
\end{aligned}
$$

Here $P$ is some constant. For the other values of $l$ and $k$ we have $p(l, k)=0$. 


\subsection{Concentration}

Theorem 2.8. Let $\delta>0$ and $k=O\left(n^{1 /(2+a+\delta)}\right)$. Then for some $\epsilon>0$, we have

$$
\mathrm{P}\left(\left|Y_{n}(k)-\mathrm{E}\left(Y_{n}(k)\right)\right|>\left(\mathrm{E}\left(Y_{n}(k)\right)\right)^{1-\epsilon}\right)=\bar{o}(1) .
$$

This is a concentration result, which means that the distribution of second degrees, as a distribution of degrees, obeys (asymptotically) a power law.

This theorem is a nontrivial application of Talagrand's inequality (see [Talagrand 96]). Instead of Talagrand's inequality it is possible to apply Azuma's inequality (see [Alon and Spencer 02]), but (as we show later) the result would have been weaker with Azuma's inequality.

We can prove an analogous result for the value $X_{n}(k)$.

Theorem 2.9. Let $\delta>0$ and $k=O\left(n^{1 /(4+a+\delta)}\right)$. Then for some $\epsilon>0$, we have

$$
\mathrm{P}\left(\left|X_{n}(k)-\mathrm{E}\left(X_{n}(k)\right)\right|>\left(\mathrm{E}\left(X_{n}(k)\right)\right)^{1-\epsilon}\right)=\bar{o}(1) .
$$

If we substitute $a=1$ in the Buckley-Osthus model, then we obtain the Bollobás-Riordan model [Bollobás and Riordan 04]. The second degrees in this model were considered in [Ostroumova and Grechnikov 12]. The concentration of second degrees in [Ostroumova and Grechnikov 12] was proved using Azuma's inequality. This inequality provided the concentration of $X_{n}(k)$ around its expectation for all $k=O\left(n^{1 /(6+\delta)}\right)$ (with any positive $\delta$ ). As stated in Theorem 2.9, Talagrand's inequality gives a stronger result: for the Bollobás-Riordan model we obtain the concentration for all $k=O\left(n^{1 /(5+\delta)}\right)$. We obtain this improvement in spite of the fact that the proof of the concentration of $X_{n}(k)$ in Theorem 2.9 uses the concentration of $Y_{n}(k)$ from Theorem 2.8, so it is not optimal in this sense.

It is possible to generalize Theorem 2.8 (and also Theorem 2.9) to the case of arbitrary $m>1$. The only problem in this case is that we have been unable to prove an analogue of Theorem 2.1 (or Corollary 2.2) for $m>1$, since it requires even more calculations. But one would expect that the following conjecture is true.

Conjecture 2.10. For every $m>1$ and $k=O\left(n^{\min \left\{\frac{1}{2+a}, \frac{1}{2 a}\right\}}\right)$, we have $\mathrm{E} Y_{n}^{m}(k)=$ $\Theta\left(\frac{n}{k^{a}}\right)$, where $Y_{n}^{m}(k)$ is the number of $k$-vertices in $H_{a, m}^{n}$.

We can generalize Theorem 2.8 in the following way. 
Theorem 2.11. Suppose Conjecture 2.10 is true. Let $m \in \mathbb{N}, \delta>0$, and $k=$ $O\left(n^{\min \left\{\frac{1}{2+a+\delta}, \frac{1}{2 a+\delta}\right\}}\right)$. Then for some $\epsilon>0$, we have

$$
\mathrm{P}\left(\left|Y_{n}^{m}(k)-\mathrm{E}\left(Y_{n}^{m}(k)\right)\right|>\left(\mathrm{E}\left(Y_{n}^{m}(k)\right)\right)^{1-\epsilon}\right)=\bar{o}(1) .
$$

In Sections 3.1-3.4, we prove Theorem 2.8 (using Corollary 2.2). In Section 3.5, we prove Theorem 2.9 using Corollary 2.4. In Section 3.6, we present a sketch of the proof of Theorem 2.11. In Section 4, we prove results from Section 2.2 (Theorems 2.1 and 2.5 and Lemma 2.7 in Sections 4.2, 4.1, and 4.3 respectively). Finally, we prove Theorem 2.3 in Section 4.4 .

\section{Concentration}

\section{I. Interpretation of the Buckley-Osthus Model in Terms of Independent Variables}

We consider the following sequence:

$$
1, \xi_{1}, 2, \xi_{2}, \ldots, n, \xi_{n},
$$

where $\xi_{1}, \ldots, \xi_{n}$ are mutually independent random variables. For every $i$, we have $\xi_{i}: \Omega_{i} \rightarrow\{1, \ldots, 2 i-1\}$ (here $\left(\Omega_{i}, \mathcal{F}_{i}, \mathrm{P}_{i}\right)$ is some probability space) and

$$
\begin{aligned}
\mathrm{P}_{i}\left(\xi_{i}=2 j-1\right) & =\frac{a}{(a+1) i-1} \quad \forall j=1, \ldots, i, \\
\mathrm{P}_{i}\left(\xi_{i}=2 j\right) & =\frac{1}{(a+1) i-1} \quad \forall j=1, \ldots, i-1 .
\end{aligned}
$$

We can interpret the sequence in the following way. Each $i$ is a vertex of a graph. Each $\xi_{i}$ is an endpoint of the edge that goes from the vertex $i$. If $\xi_{i}=2 j-1$, then the edge goes to the vertex $j$. If $\xi_{i}=2 j$, then we say that the edge from the vertex $i$ goes to the same vertex as the edge from the vertex $j$. The value of the variable $\xi_{j}$ can also be even (say $\xi_{j}=2 j_{1}$ for some integer $j_{1}$ ). Then the edge from the vertex $i$ is again redirected according to the variable $\xi_{j_{1}}$. Finally, this process stops at some odd value $2 v-1$, and we say that $\xi_{i}$ (as well as $\xi_{j}$ and $\xi_{j_{1}}$ ) leads to the vertex $v$. We also say that $\xi_{i}$ leads to $\xi_{j}$.

It is not hard to check that the graph model we have obtained is exactly the Buckley-Osthus model. Indeed, at each time step $i$, the in-degree of each vertex $j \in\{1, \ldots, i-1\}$ is equal to the number of variables that lead (directly or indirectly) to the vertex $j$.

Let us give yet another interpretation of the model described above. Consider a vertex $v$ from the obtained graph. We can think of all the variables that lead to $v$ 
as a rooted tree, with $v$ as the root. Let $X=\left\{\xi_{i_{1}}, \ldots, \xi_{i_{d}}\right\}$ be the set of variables that lead to $v$. We inductively construct the corresponding tree on $d$ vertices $i_{1}, \ldots, i_{d}$. First consider the variables $\xi_{i_{1}^{1}}, \ldots, \xi_{i_{l_{1}}}$ from $X$ that lead to $v$ directly. The corresponding vertices $i_{1}^{1}, \ldots, i_{l_{1}}^{1}$ are adjacent to $v$ in the tree. Suppose we choose all the vertices at distance $\leq s$ from $v$ and suppose that $i_{1}^{s}, \ldots, i_{l_{s}}^{s}$ are the vertices at distance $s$ from $v$. Consider the set $\left\{\xi_{i_{1}^{s+1}}, \ldots, \xi_{i_{l_{s+1}^{s+1}}}\right\}$ of variables that lead to some of $\xi_{i_{1}^{s}}, \ldots, \xi_{i_{1 s}^{s}}$. We join each of the vertices $i_{1}^{s+1}, \ldots, i_{l_{s+1}+1}^{s}$ to the corresponding vertex from $\left\{i_{1}^{s}, \ldots, i_{l_{s}}^{s}\right\}$. We thus obtain the set of vertices at distance $s+1$ from $v$.

\subsection{Decreasing the Number of $k$-Vertices}

We fix a value $\mathbf{x}=\left(x_{1}, \ldots, x_{n}\right)$ of the random vector $\xi=\left(\xi_{1}, \ldots, \xi_{n}\right)$ from the probability space $\Omega=\prod_{i=1}^{n} \Omega_{i}$. The quantity $Y_{n}(k)$ is a function from $\Omega$ to $\mathbb{N}$. We discuss the following question. How can the value $Y_{n}(k)=Y_{n}(k, \mathbf{x})$ decrease if we change one coordinate $x_{i}$ of the vector $\mathbf{x}$ ? In other words, we want to find $c(i, \mathbf{x})=\max _{\mathbf{x}^{\prime}}\left(Y_{n}(k, \mathbf{x})-Y_{n}\left(k, \mathbf{x}^{\prime}\right)\right)$, where $\mathbf{x}^{\prime}$ is an arbitrary vector that differs from $\mathbf{x}$ in exactly the $i$ th coordinate.

Lemma 3.I. For every $\mathbf{x}=\left(x_{1}, \ldots, x_{n}\right)$ and $i \in\{1, \ldots, n\}$, we have $c(i, \mathbf{x}) \leq 2 k+1$.

Proof. It is convenient to think about the tree interpretation of the random variables. If we change a value $x_{i}$ of one random variable $\xi_{i}$ to some value $x_{i}^{\prime}$, then all the variables that lead to $x_{i}$ are redirected to $x_{i}^{\prime}$. In terms of the tree interpretation, we pick the branch of the tree in which all the edges lead to $x_{i}$ : if $x_{i}^{\prime}$ is odd, then we link the branch to the vertex with the number $\left(x_{i}^{\prime}+1\right) / 2$; if $x_{i}^{\prime}$ is even, then we link the branch to the variable $\xi_{x_{i}^{\prime} / 2}$.

We want to interpret the change of one coordinate in terms of the graph $H_{a, 1}^{n}$. Suppose $x_{i}$ leads to a vertex $v$. Then all the variables that lead to $x_{i}$ lead to $v$. If we change $x_{i}$ to $x_{i}^{\prime}$ and $x_{i}^{\prime}$ leads to $v^{\prime}$, then we change the value of all such variables from $v$ to $v^{\prime}$. Or in terms of $H_{a, 1}^{n}$, we take a bundle of edges in the vertex $v$ and move the bundle to the vertex $v^{\prime}$. More precisely, if we have a bundle of edges $\left(i_{1}, v\right), \ldots,\left(i_{d}, v\right)$, then after the change, we have the edges $\left(i_{1}, v^{\prime}\right), \ldots,\left(i_{d}, v^{\prime}\right)$. All the rest stays the same.

Now we proceed to the proof. We must show that after the change of the $i$ th coordinate, the number of $k$-vertices we spoil does not exceed $2 k+1$. Suppose we moved a bundle of edges $\left(i_{1}, v\right), \ldots,\left(i_{d}, v\right)$. It is easy to see that we could spoil only the $k$-vertices that have a common edge with $v$ or $v$ itself. Note that we could not spoil the $k$-vertices in a neighborhood of $v^{\prime}$. 
We split the set $N_{v}$ of the vertices incident to $v$ into two parts: $I=\bigcup_{j=1}^{d}\left\{i_{j}\right\}$ and $N_{v} \backslash I$. If $\left|N_{v} \backslash I\right| \geq k+1$, then after changing the edges from the bundle, all the $k$-vertices from $N_{v} \backslash I$ are still $k$-vertices. Indeed, all the edges in vertex $v$ except for one are 2-incident edges for any neighbor of $v$, so there are at least $k$ such edges for every vertex from $N_{v} \backslash I$. Similarly, if $|I| \geq k+1$, then no $k$ vertices among $i_{1}, \ldots, i_{d}$ are spoiled except for at most one, since they are all adjacent to the vertex $v^{\prime}$. The only case in which some of $i_{1}, \ldots, i_{d}$ are spoiled is $i_{j}=v^{\prime}$, and so we will not count the edges $\left(i_{1}, v^{\prime}\right), \ldots,\left(i_{d}, v^{\prime}\right)$ in the second degree of $i_{j}$.

Finally, the number of $k$-vertices we spoil does not exceed

$$
\min \{|I|, k\}+\min \left\{\left|N_{v} \backslash I\right|, k\right\}+1 \leq 2 k+1 .
$$

This completes the proof.

We now want to estimate the influence of each variable more accurately. Suppose $Y_{n}(k, \mathbf{x})=q$. For each $k$-vertex $v_{j}, j=1, \ldots, q$, we consider a subset of coordinates $K_{j}=K_{v_{j}}(\mathbf{x})=\left\{i_{1}^{j}, \ldots, i_{d_{j}}^{j}\right\}$ such that $v_{j}$ is a $k$-vertex for every $\mathbf{y}$ that agrees with $\mathbf{x}$ on the coordinates from $K_{j}$. It is worth noting that $K_{j}$ depends on $\mathbf{x}$, but is not uniquely defined by it. For any choice of the sets $K_{1}, \ldots, K_{q}$, we denote their collection by $\mathcal{K}=\mathcal{K}(\mathbf{x})$. Clearly, $Y_{n}(k, \mathbf{y}) \geq q$, for any $\mathbf{y}$ that agrees with $\mathbf{x}$ on all the coordinates from all $K_{j} \in \mathcal{K}$.

For each coordinate $i$, we define its multiplicity $C(i, \mathbf{x}, \mathcal{K})=\left|\left\{j: i \in K_{j}\right\}\right|$. It is easy to see that for any $\mathbf{x}$ and any $\mathcal{K}$ one has $c(i, \mathbf{x}) \leq C(i, \mathbf{x}, \mathcal{K})$. So we have

$$
c(i, \mathbf{x}) \leq \min \{2 k+1, C(i, \mathbf{x}, \mathcal{K})\}=: c_{i}(\mathbf{x}, \mathcal{K}) .
$$

We call a collection $\mathcal{K}$ stable if for every $k$-vertex $v_{i}$, we construct all the sets $K_{i}$ according to the following rule: if $K_{i}$ contains some of the coordinates that lead to a vertex $w$, then $K_{i}$ contains all of the coordinates that lead to $w$.

It should not be surprising that for such systems of sets we can prove an analogue of Lemma 3.1. Namely, consider a vector $\mathbf{x}$, the corresponding $k$-vertices $v_{j}, j=1, \ldots, q$, and some stable collection $\mathcal{K}$. Let $i \in\{1, \ldots, n\}$ and $\mathcal{K} \backslash\{i\}:=$ $\left\{K_{j} \backslash\{i\}, j=1, \ldots, q\right\}$. Given $i$, there exist at least $q-c_{i}(\mathbf{x}, \mathcal{K})$ such $k$-vertices that are $k$-vertices for every $\mathbf{x}^{\prime}$ with $x_{s}^{\prime}=x_{s}$ for all $s \in K_{j} \backslash\{i\}, j=1, \ldots, q$. To prove this fact one has to follow the proof of Lemma 3.1 and make sure that the proof works also for this case. The only additional consideration needed is that the number of $k$-vertices we lose does not exceed the multiplicity $C(j, \mathbf{x}, \mathcal{K})$. 
Lemma 3.2. Let $\mathcal{K}$ be a stable collection as described above, and let $J$ be any subset of $\{1, \ldots, n\}$. We have $Y_{n}(k, \mathbf{x})-Y_{n}\left(k, \mathbf{x}^{\prime}\right) \leq \sum_{j \in J} c_{j}(\mathbf{x}, \mathcal{K})$ for every vector $\mathbf{x}^{\prime}$ such that $x_{i}^{\prime}=x_{i}$ for all $i \in\{1, \ldots, n\} \backslash J$.

Proof. Suppose we change one coordinate $j$ of $\mathbf{x}$ and obtain some vector $\hat{\mathbf{x}}$. Then we consider $d_{j}:=C(j, \mathbf{x}, \mathcal{K}) k$-vertices $w_{1}, \ldots, w_{d_{j}}$ such that $j \in K_{w_{i}}(\mathbf{x})$. We remove $j$ from each of these sets and we check for each $i=1, \ldots, d_{j}$ whether the obtained collection guarantees that $w_{i}$ is a $k$-vertex. If $w_{i}$ is a $k$-vertex then we define $K_{w_{i}}(\hat{\mathbf{x}})=K_{w_{i}}(\mathbf{x}) \backslash\{j\}$. If $w_{i}$ is not a $k$-vertex, then we exclude the set $K_{w_{i}}(\mathbf{x})$ from $\mathcal{K}$. At the end of this step we obtain a new collection $\hat{\mathcal{K}}$. To prove the lemma, we need one consideration. Namely, instead of changing the edges $\left(i_{1}, v\right), \ldots,\left(i_{d}, v\right)$ to $\left(i_{1}, v^{\prime}\right), \ldots,\left(i_{d}, v^{\prime}\right)$, we can create a new imaginary vertex $w$ and change the edges to $\left(i_{1}, w\right), \ldots,\left(i_{d}, w\right)$. We denote the obtained graph by $G_{w}$. We do not count $w$ as a $k$-vertex even if it has $\geq k 2$-incident edges. It is easy to check that for this graph, the collection $\hat{\mathcal{K}}$ is stable.

The number of $k$-vertices (except for $w$ ) in the graph $G_{w}$ is definitely not greater than the same number for the graph corresponding to $\hat{\mathbf{x}}$. Moreover, the multiplicity of each coordinate in $\hat{\mathcal{K}}$ is less than or equal to the corresponding multiplicity in $\mathcal{K}$. We also have $Y_{n}(k, \mathbf{x})-Y_{n}\left(k, G_{w}\right) \leq c_{j}(\mathbf{x}, \mathcal{K})$. Similarly, if $\mathbf{x}^{\prime}$ differs from $\mathbf{x}$ in $l$ coordinates, then the graph corresponding to $\mathbf{x}^{\prime}$ has at least as many $k$-vertices as the graph $G$ obtained by forming $l$ imaginary vertices. Moreover, at each step (if we change the coordinate $j^{\prime}$ and form the corresponding graph $\left.G^{\prime}\right)$ we spoil at most $\min \left\{2 k+1, C\left(j^{\prime}, \mathbf{x}, \mathcal{K}\right)\right\} k$-vertices and obtain a stable system of sets.

Consequently, we have

$$
Y_{n}(k, \mathbf{x})-Y_{n}\left(k, \mathbf{x}^{\prime}\right) \leq Y_{n}(k, \mathbf{x})-Y_{n}(k, G) \leq \sum_{j \in J} c_{j}(\mathbf{x}, \mathcal{K}),
$$

and the proof is complete.

\subsection{Construction of a Suitable Set $\mathcal{K}$}

Lemma 3.3. Suppose $Y_{n}(k, \mathbf{x})=q$ for some vector $\mathbf{x}$ in the corresponding graph $G_{\mathbf{X}}$. Then we can construct a stable system of sets $\mathcal{K}=\left\{K_{1}, \ldots, K_{q}\right\}$ such that $\sum_{i=1}^{n} c_{i}(\mathbf{x}, \mathcal{K}) \leq(4 k+5) q$

Proof. First consider the set $V$ of vertices with degree at least $k+2$. Put $N V=\{u: u$ is a neighbor of $v \in V\}$. Note that a vertex from $V$ can also belong to $N V$. Assume that $|N V|=z$. All vertices from $N V$ are $k$-vertices. Let $B V$ be the set of vertices from $N V$ that do not have an outgoing edge that goes 
to $V$. We have $|B V| \leq z /(k+1)$, since each vertex has at most one outgoing edge.

We denote by $L_{v}, L_{v} \subset\{1, \ldots, n\}$ the set of coordinates that lead to $v$. We also put $L V=\cup_{v \in V} L_{v}$ and $L B V=\cup_{v \in B V} L_{v}$. For every $u \in N V$, we put $K_{u}=$ $L V \cup L B V$. It is easy to see that for every $\mathbf{x}^{\prime}$ such that $x_{i}=x_{i}^{\prime}$ for every $i \in$ $L V \cup L B V$, the vertex $u$ is a $k$-vertex in the graph corresponding to $\mathbf{x}^{\prime}$.

For $i \in L V \cup L B V$ we estimate $c_{i}(\mathbf{x}, \mathcal{K})$ by $2 k+1$. Note that $|L V| \leq z+z / k$. We add additional $z / k$ variables because the vertices from $V$ can have loops. We have $\operatorname{deg} w \leq k+1$ for $w \in B V \backslash V$ and $|B V \backslash V| \leq z /(k+1)$. Therefore, $|L B V \backslash L V| \leq z$. So we have

$$
\begin{aligned}
\sum_{i \in L V \cup L B V} c_{i}(\mathbf{x}, \mathcal{K}) & \leq(2 k+1)(|L B V \backslash L V|+|L V|) \leq(2 k+1)\left(2 z+\frac{z}{k}\right) \\
& \leq(4 k+5) z .
\end{aligned}
$$

Next we consider the set $W$ of the remaining $k$-vertices. We have $|W|=q-z$. By definition, for every $w \in W$, all the neighbors $N_{w}$ of $w$ have degree less than or equal to $k+1$.

For each $w \in W$ we consider $V_{w}=\left\{v_{1}, \ldots, v_{w}\right\}, V_{w} \subset N_{w}$, such that the number of edges adjacent to at least one of $v_{i} \in N_{w}$ and not adjacent to $w$ is between $k$ and $2 k$. We can find such $V_{w}$, since $w$ is a $k$-vertex. We can choose $v_{i} \in N_{w}$ one by one until the total number of 2 -adjacent edges does not exceed $k$. But it cannot exceed $2 k, \operatorname{since} \operatorname{deg} v_{i} \leq k+1$ for $v_{i} \in N_{w}$. Denote by $L V_{w}$ all the variables that lead to $V_{w}$.

Now for each $w \in W$, we put $K_{w}=L V_{w} \cup L_{w}$. Note that $\left|L V_{w}\right| \leq 2 k$, and for $w \in W \backslash V$ we have $\left|L_{w}\right| \leq k+1$.

Now we can make the final estimate:

$$
\begin{aligned}
\sum_{i=1}^{n} c_{i}(\mathbf{x}, \mathcal{K})= & \sum_{i \in L V \cup L B V} c_{i}(\mathbf{x}, \mathcal{K})+\sum_{i \in\{1, \ldots, n\} \backslash L V \cup L B V} c_{i}(\mathbf{x}, \mathcal{K}) \leq(4 k+5) z \\
& +\sum_{w \in W}\left|L V_{w}\right|+\sum_{w \in W \backslash V}\left|L_{w}\right| \\
\leq & (4 k+5) z+2 k(q-z)+(k+1)(q-z) \leq(4 k+5) q .
\end{aligned}
$$

The fact that $\mathcal{K}$ is a stable system of sets follows from the construction.

\subsection{Application of Talagrand's Inequality}

First we briefly review Talagrand's inequality (see, e.g., [Alon and Spencer 02]). Let $\Omega=\prod_{i=1}^{n} \Omega_{i}$ be a product probability space with product measure. Suppose 
$\alpha=\left(\alpha_{1}, \ldots, \alpha_{n}\right), \sum_{i=1}^{n} \alpha_{i}^{2}=1$. We define the following distance between a set $A \subset \Omega$ and a point $\mathrm{x} \in \Omega$ :

$$
\operatorname{dist}(A, \mathbf{x})=\max _{\alpha} \min _{\mathbf{y} \in A} \sum_{i \in I_{\mathbf{x y}}} \alpha_{i},
$$

where $I_{\mathrm{xy}}=\left\{i: x_{i} \neq y_{i}\right\}$.

For $t>0$ we denote by $A_{t}$ the set $\{\mathbf{x}: \operatorname{dist}(A, \mathbf{x}) \leq t\}$.

Theorem 3.4. (Talagrand's inequality.) For every $t>0$ and $A \subset \Omega$, we have

$$
\mathrm{P}(A)\left(1-\mathrm{P}\left(A_{t}\right)\right) \leq e^{-t^{2} / 4} \text {. }
$$

We use this inequality to derive the following theorem.

Theorem 3.5. For $t>0, k, s \in \mathbb{N}$, and $f(s)$ that satisfies the condition $f^{2}(s)>$ $(2 k+1)(4 k+5) s$, we have $\mathrm{P}\left(Y_{n}(k) \leq s-t f(s)\right) \mathrm{P}\left(Y_{n}(k) \geq s\right) \leq e^{-t^{2} / 4}$.

Proof. The inequality is trivial for $t f(s)>s$, so we can assume without loss of generality that $t f(s) \leq s$. Since $\xi_{i}$ are independent, we can apply Talagrand's inequality to the points $\mathbf{x}$ from the probability space $\Omega$. In fact, all we need to prove is that for every $\mathbf{x}$ such that $Y_{n}(k, \mathbf{x}) \geq s$, we have $\mathbf{x} \notin A_{t}$, where $A=$ $\left\{\mathbf{y}: Y_{n}(k, \mathbf{y}) \leq s-t f(s)\right\}$.

Suppose $Y_{n}(k, \mathbf{x})=q \geq s$. Given $\mathbf{x}$, we fix a system of sets $\mathcal{K}$ as in Lemma 3.3. Then by Lemma 3.2, for every $\mathbf{y} \in A$ we have $q-s+t f(s) \leq Y_{n}(k, \mathbf{x})-$ $Y_{n}(k, \mathbf{y}) \leq \sum_{j \in I_{\mathbf{x y}}} c_{j}(\mathbf{x}, \mathcal{K})$.

We define a suitable vector $\alpha=\alpha(\mathbf{x})$. Namely,

$$
\alpha_{i}=\frac{c_{i}(\mathbf{x}, \mathcal{K})}{\sqrt{\sum_{j=1}^{n} c_{j}^{2}(\mathbf{x}, \mathcal{K})}} .
$$

It is easy to see that $\sum \alpha_{j}^{2}=1$.

We have

$$
\sum_{j=1}^{n} c_{j}^{2}(\mathbf{x}, \mathcal{K}) \leq \max _{j} c_{j}(\mathbf{x}, \mathcal{K}) \sum_{j=1}^{n} c_{j}(\mathbf{x}, \mathcal{K}) \leq(2 k+1)(4 k+5) q .
$$

In the last inequality we used Lemma 3.3 and the definition of $c_{j}(\mathbf{x}, \mathcal{K})$.

Now we show that $\sum_{i \in I_{\mathbf{x y}}} \alpha_{i}>t$ for every $\mathbf{y} \in A$ :

$$
\sum_{i \in I_{\mathbf{x y}}} \alpha_{i}=\frac{\sum_{i \in I_{\mathbf{x y}}} c_{i}(\mathbf{x}, \mathcal{K})}{\sqrt{\sum_{j=1}^{n} c_{j}^{2}(\mathbf{x}, \mathcal{K})}} \geq \frac{q-s+t f(s)}{\sqrt{(2 k+1)(4 k+5) q}} \geq \frac{t f(s)}{\sqrt{(2 k+1)(4 k+5) s}}>t .
$$


The second inequality holds because for $q \geq s, t f(s) \leq s$, we have

$$
\frac{q-s+t f(s)}{q} \geq \frac{t f(s)}{s} \text {. }
$$

The last inequality follows from the statement of the theorem.

From (3.1) we obtain that $\operatorname{dist}(A, \mathbf{x})>t$, in other words, $\mathbf{x} \notin A_{t}$.

We apply Theorem 3.5 with $t=2 \ln n, s=m\left(Y_{n}(k)\right)+t\left(E Y_{n}(k)\right)^{1-\varepsilon}, f(s)=$ $\left(\mathrm{E} Y_{n}(k)\right)^{1-\varepsilon}$. Here $m\left(Y_{n}(k)\right)$ is the median of $Y_{n}(k)$, and consequently, $\mathrm{P}\left(Y_{n}(k) \leq\right.$ $s-t f(s)) \geq 1 / 2$. Since for any random variable $Z$ we have $m(Z) \leq 2 \mathrm{E} Z$, it is easy to see that the conditions of Theorem 3.5 hold if

$$
\left(\mathrm{E} Y_{n}(k)\right)^{1-2 \varepsilon} \geq 12(2 k+1)^{2} \ln n .
$$

If $\varepsilon$ is small enough, then this inequality is a consequence of Corollary 2.2 and the conditions of Theorem 2.8.

We obtain that

$$
\mathrm{P}\left(Y_{n}(k) \geq m\left(Y_{n}(k)\right)+2 \ln n\left(\mathrm{E} Y_{n}(k)\right)^{1-\varepsilon}\right) \leq 2 e^{-t^{2} / 4}=\bar{o}(1 / n),
$$

and since $Y_{n}(k) \leq n$ for all $k$, we have

$$
\mathrm{E} Y_{n}(k) \leq m\left(Y_{n}(k)\right)+2 \ln n\left(\mathrm{E} Y_{n}(k)\right)^{1-\varepsilon}+\bar{o}(1) .
$$

Similarly, we can derive that

$$
\mathrm{P}\left(Y_{n}(k) \leq m\left(Y_{n}(k)\right)-2 \ln n\left(\mathrm{E} Y_{n}(k)\right)^{1-\varepsilon}\right) \leq 2 e^{-t^{2} / 4}=\bar{o}(1 / n)
$$

and

$$
\mathrm{E} Y_{n}(k) \geq m\left(Y_{n}(k)\right)-2 \ln n\left(\mathrm{E} Y_{n}(k)\right)^{1-\varepsilon}-\bar{o}(1) .
$$

Consequently, for some $\delta>0$ and all sufficiently large $n$, we have $\mid \mathrm{E} Y_{n}(k)-$ $m\left(Y_{n}(k)\right) \mid \leq\left(\mathrm{E} Y_{n}(k)\right)^{1-\delta}$. Therefore, for some $\varepsilon^{\prime}>0$,

$$
\mathrm{P}\left(\left|Y_{n}(k)-\mathrm{E}\left(Y_{n}(k)\right)\right|>\mathrm{E}\left(Y_{n}(k)\right)^{1-\varepsilon^{\prime}}\right)=\bar{o}(1) .
$$

This concludes the proof of Theorem 2.8.

\subsection{Proof of Theorem 2.9}

We use the obvious fact that $X_{n}(k)=Y_{n}(k)-Y_{n}(k+1)$. Fix some $\epsilon^{\prime}>0$. First we want to apply Theorem 3.5 to $Y_{n}(k)$ and $Y_{n}(k+1)$. We argue as after the proof of Theorem 3.5. We put $f(s)=n^{1-\epsilon^{\prime}} / k^{1+a}, t=2 \ln n$, and $s_{1}=m\left(Y_{n}(k)\right)+$ $t f(s), s_{2}=m\left(Y_{n}(k)\right), s_{3}=m\left(Y_{n}(k+1)\right)+t f(s), s_{4}=m\left(Y_{n}(k+1)\right)$. 
We apply Theorem 3.5 to $Y_{n}(k)$ with $s_{1}$ and $s_{2}$, and to $Y_{n}(k+1)$ with $s_{3}$ and $s_{4}$, and obtain

$$
\begin{array}{r}
\mathrm{P}\left(\left|Y_{n}(k)-\mathrm{E}\left(Y_{n}(k)\right)\right|>\frac{n^{1-\epsilon^{\prime}+\bar{o}(1)}}{k^{1+a}}\right)=\bar{o}(1), \\
\mathrm{P}\left(\left|Y_{n}(k+1)-\mathrm{E}\left(Y_{n}(k+1)\right)\right|>\frac{n^{1-\epsilon^{\prime}+\bar{o}(1)}}{k^{1+a}}\right)=\bar{o}(1),
\end{array}
$$

provided

$$
\frac{n^{2-2 \epsilon^{\prime}}}{k^{2+2 a}} \geq \Theta\left(n k^{2-a}\right) \ln n .
$$

It is easy to see that this holds if the conditions of Theorem 2.9 are satisfied for some $\delta>0$. We have $\left|X_{n}(k)-\mathrm{E}\left(X_{n}(k)\right)\right| \leq\left|Y_{n}(k)-\mathrm{E}\left(Y_{n}(k)\right)\right|+\mid Y_{n}(k+$ 1) $-\mathrm{E}\left(Y_{n}(k+1)\right) \mid$, so

$$
\mathrm{P}\left(\left|X_{n}(k)-\mathrm{E}\left(X_{n}(k)\right)\right|>\frac{n^{1-\epsilon^{\prime}+\bar{o}(1)}}{k^{1+a}}\right)=\bar{o}(1) .
$$

Since $n^{1-\epsilon^{\prime}+\bar{o}(1)} / k^{1+a}=\mathrm{E}\left(X_{n}(k)\right)^{1-\epsilon}$ for some $\epsilon>0$, this inequality completes the proof of Theorem 2.9 .

\subsection{Generalization to the Case of Arbitrary $m$}

The proof of Theorem 3.5 can be modified to the case of the graph $H_{a, m}^{n}$. We present only a sketch of the argument. Suppose $m>1$ is fixed. The number of variables changes from $n$ to $m n$. The interpretation in terms of independent variables works for this case. Lemmas 3.1, 3.2, 3.3 hold for $m>1$, but with some minor changes.

When we take a bundle of edges from a vertex $v$ and move it to some vertex $v^{\prime}$, we can spoil not only the neighborhood of $v$, but also the vertex $v^{\prime}$. Namely, suppose we change edges $\left(v, w_{1}\right), \ldots,\left(v, w_{l}\right)$ to $\left(v^{\prime}, w_{1}\right), \ldots,\left(v^{\prime}, w_{l}\right)$. If $v^{\prime}$ was a $k$-vertex and edges $\left(v, w_{1}\right), \ldots,\left(v, w_{l}\right)$ were counted in the second degree of $v^{\prime}$, then the second degree of $v^{\prime}$ may decrease (this is impossible in the graph $H_{a, 1}^{n}$, since $H_{a, 1}^{n}$ is a tree). It is not difficult to see that we cannot spoil the other vertices.

Hence, we can formulate some analogues of Lemmas 3.1 and 3.2.

Lemma 3.6. For every $\mathbf{x}=\left(x_{1}, \ldots, x_{n}\right)$ and $i \in\{1, \ldots, n\}$, we have $c(i, \mathbf{x}) \leq 2 k+2$. 
We also have

$$
c(i, \mathbf{x})-1 \leq \min \{2 k+1, C(i, \mathbf{x}, \mathcal{K})\}=: c_{i}(\mathbf{x}, \mathcal{K}) .
$$

Lemma 3.7. Let $\mathcal{K}$ be a stable collection of sets. We have $Y_{n}(k, \mathbf{x})-Y_{n}\left(k, \mathbf{x}^{\prime}\right) \leq$ $\sum_{j \in J}\left(c_{j}(\mathbf{x}, \mathcal{K})+1\right)$ for every vector $\mathbf{x}^{\prime}$ such that $x_{i}^{\prime}=x_{i}$ for all $i \in\{1, \ldots, n\} \backslash J$.

Lemma 3.3 holds for $c_{j}(\mathbf{x}, \mathcal{K})$ and $m>1$ without any changes.

The only thing left is to modify the proof of Theorem 3.5. We put

$$
\alpha_{i}=\frac{c_{i}(\mathbf{x}, \mathcal{K})+1}{\sqrt{\sum_{j=1}^{m n}\left(c_{j}(\mathbf{x}, \mathcal{K})+1\right)^{2}}} .
$$

Then

$$
\begin{aligned}
\sum_{j=1}^{m n}\left(c_{j}(\mathbf{x}, \mathcal{K})+1\right)^{2} & \leq\left(\max _{j} c_{j}(\mathbf{x}, \mathcal{K})+2\right) \sum_{j=1}^{m n} c_{j}(\mathbf{x}, \mathcal{K})+m n \\
& \leq(2 k+3)(4 k+5) q+m n
\end{aligned}
$$

Finally,

$$
\begin{aligned}
\sum_{i \in I_{\mathrm{xy}}} \alpha_{i} & =\frac{\sum_{i \in I_{\mathrm{xy}}}\left(c_{i}(\mathbf{x}, \mathcal{K})+1\right)}{\sqrt{\sum_{j=1}^{m n}\left(c_{j}(\mathbf{x}, \mathcal{K})+1\right)^{2}}} \geq \frac{q-s+t f(s)}{\sqrt{(2 k+3)(4 k+5) q+m n}} \\
& \geq \frac{t f(s)}{\sqrt{(2 k+3)(4 k+5) s+m n}}>t
\end{aligned}
$$

if $f^{2}(s)>(2 k+3)(4 k+5) s+m n$. So we can formulate an analogue of Theorem 3.5 .

Theorem 3.8. For $t>0, m, k, s \in \mathbb{N}$, and $f(s)$ that satisfies the condition $f^{2}(s)>$ $(2 k+3)(4 k+5) s+m n$, we have

$$
\mathrm{P}\left(Y_{n}^{m}(k) \leq s-t f(s)\right) \mathrm{P}\left(Y_{n}^{m}(k) \geq s\right) \leq e^{-t^{2} / 4} .
$$

Finally, arguing as following the proof of Theorem 3.5, one can see that Theorem 2.11 follows from Theorem 3.8 and Conjecture 2.10.

\section{Estimation of $E Y_{n}(k)$}

We need the following notation. Let $X$ be a function on $n$ (the number of vertices), $l$ (the first degree we are interested in), $k$ (the second degree we are interested in); then denote by $\theta(X)$ some function on $n, l, k$ such that $|\theta(X)|<X$. 


\section{I. Proof of Theorem 2.5}

It follows from the definition of $H_{a, 1}^{n}$ that $N_{n}(l, 0)=N_{n}(0, k)=0$. Indeed, since we have no vertices of degree 0 , we see that $N_{n}(0, k)=0$. Since vertices with loops are not counted in $N_{n}(l, k)$, we have no vertices of second degree 0 , and $N_{n}(l, 0)=0$. Therefore, we have $\mathrm{E} N_{n}(l, 0)=\mathrm{E} N_{n}(0, k)=0$. We want to prove that there exists a constant $C$ such that

$$
\mathrm{E} N_{n}(l, k)=c(l, k)(n+\theta(n, l, k)),
$$

where $|\theta(n, l, k)|<C(l+k)^{1+a}$.

Let us demonstrate that $\mathrm{E} N_{n}(1, k)=c(1, k)\left(n+\theta\left(C k^{1+a}\right)\right)$. We shall use induction on $k$. For $k=0$, there is nothing to prove.

Assume that for $j<k$ and for all $n$ we have

$$
\mathrm{E} N_{n}(1, j)=c(1, j)\left(n+\theta\left(C j^{1+a}\right)\right) .
$$

Denote by $N_{n}(l)$ the number of vertices with degree $l$ in $H_{a, 1}^{n}$. To prove that $\mathrm{E} N_{n}(1, k)=c(1, k)\left(n+\theta\left(C k^{1+a}\right)\right)$, we use induction on the number of vertices and the equality

$$
\begin{aligned}
& \mathrm{E}\left(N_{i+1}(1, k) \mid N_{i}(1, k), N_{i}(1, k-1), N_{i}(k)\right) \\
& \quad=N_{i}(1, k)\left(1-\frac{k+2 a}{(a+1) i+a}\right)+\frac{(k-1+a) N_{i}(1, k-1)}{(a+1) i+a}+\frac{(k-1+a) N_{i}(k)}{(a+1) i+a} .
\end{aligned}
$$

Let us explain this equality. Suppose we have constructed $H_{a, 1}^{i}$. We add one vertex and one edge. There are $N_{i}(1, k)$ vertices with degree 1 and with second degree $k$ in $H_{a, 1}^{i}$. The probability that we "spoil" one of these vertices is $\frac{k+2 a}{(a+1) i+a}$. We also have $N_{i}(1, k-1)$ vertices with degree 1 and with second degree $k-1$. The probability that one of these vertices has degree 1 and second degree $k$ in $H_{a, 1}^{i+1}$ is $\frac{k-1+a}{(a+1) i+a}$. Finally, with probability equal to $\frac{k-1+a}{(a+1) i+a}$, the vertex $i+1$ has necessary degrees in $H_{a, 1}^{i+1}$. From (4.1) we obtain

$$
\begin{aligned}
\mathrm{E} N_{i+1}(1, k)= & \mathrm{E} N_{i}(1, k)\left(1-\frac{k+2 a}{(a+1) i+a}\right)+\frac{(k-1+a) \mathrm{E} N_{i}(1, k-1)}{(a+1) i+a} \\
& +\frac{(k-1+a) \mathrm{E} N_{i}(k)}{(a+1) i+a}
\end{aligned}
$$

Note that if we have at least one vertex with first degree 1 and second degree $k$ in $H_{a, 1}^{i}$, then we have at least $k$ edges in this graph. Therefore $\mathrm{E} N_{i}(1, k)=0$ when $i<k$. Consider the case $i=k$. First, note that

$$
\mathrm{E} N_{k}(1, k) \geq c(1, k)\left(k+\theta\left(C k^{1+a}\right)\right)
$$


with some $C$, because we can put $\theta\left(C k^{1+a}\right)=-k$ for sufficiently large $C$. For a finite number of small $k$ we can find a constant $C$ such that

$$
\mathrm{E} N_{k}(1, k)=c(1, k)\left(k+\theta\left(C k^{1+a}\right)\right) .
$$

Using (4.2), Lemma 2.6, and the assumptions of the theorem, we get

$$
\begin{aligned}
& \mathrm{E} N_{k}(1, k)=\mathrm{E} N_{k-1}(1, k-1) \frac{k-1+a}{a k+k-1}+M_{k-1}^{1}(k) \frac{k-1+a}{a k+k-1} \\
&= c(1, k-1) \frac{k-1+a}{a k+k-1}\left(k-1+\theta\left(C(k-1)^{1+a}\right)\right) \\
&+c(k) \frac{k-1+a}{a k+k-1}\left(k-1+\theta\left(C_{1} k^{1+a}\right)\right) \\
&= c(1, k) \frac{(k+3 a+1)(k-1)}{a k+k-1}+c(1, k-1) \frac{k-1+a}{a k+k-1} \theta\left(C(k-1)^{1+a}\right) \\
&+c(k) \frac{k-1+a}{a k+k-1} \theta\left(C_{1} k^{1+a}\right) \\
&= k c(1, k)+\frac{3 a k+k-3 a-1-a k^{2}}{a k+k-1} c(1, k) \\
&+c(1, k-1) \frac{k-1+a}{a k+k-1} \theta\left(C(k-1)^{1+a}\right)+c(k) \frac{k-1+a}{a k+k-1} \theta\left(C_{1} k^{1+a}\right) \\
& \leq k c(1, k)+\frac{\left(3 a k+k-3 a-1-a k^{2}\right)(a+k-1)}{(a k+k-1)(k+3 a+1)} c(1, k-1) \\
&+\frac{\left(3 a k+k-3 a-1-a k^{2}\right)(a+k-1)}{(a k+k-1)(k+3 a+1)} c(k) \\
&+c(1, k-1) \frac{C(k-1+a)}{a k+k-1}(k-1)^{1+a}+c(k) \frac{k-1+a}{a k+k-1} C_{1} k^{1+a} \\
& \leq k c(1, k)+\frac{C(a+k-1)}{k+3 a+1} c(1, k-1) k^{1+a}+\frac{C(a+k-1)}{k+3 a+1} c(k) k^{1+a} .
\end{aligned}
$$

This holds for large values of $k$. Indeed,

$$
\frac{\left(3 a k+k-3 a-1-a k^{2}\right)}{(a k+k-1)(k+3 a+1)}+\frac{C(k-1)^{1+a}}{a k+k-1} \leq \frac{C}{k+3 a+1} k^{1+a}
$$

if $k$ and $C$ are large enough. 
Consider the case $i \geq k$. Using (4.2), Lemma 2.6, and the inductive assumption, we get

$$
\begin{aligned}
\mathrm{E} N_{i+1} & (1, k)=\mathrm{E} N_{i}(1, k)\left(1-\frac{k+2 a}{(a+1) i+a}\right)+\mathrm{E} N_{i}(1, k-1) \frac{k-1+a}{(a+1) i+a} \\
& +M_{i}^{1}(k) \frac{k-1+a}{(a+1) i+a} \\
= & c(1, k)\left(i+\theta\left(C k^{1+a}\right)\right)\left(1-\frac{k+2 a}{(a+1) i+a}\right) \\
& +c(1, k-1)\left(i+\theta\left(C(k-1)^{1+a}\right)\right) \frac{k-1+a}{(a+1) i+a} \\
& +c(k)\left(i+\theta_{1}\left(C_{1} k^{1+a}\right)\right) \frac{k-1+a}{(a+1) i+a}=c(1, k)(i+1) \\
& -c(1, k) \frac{i(k+3 a+1)+a}{(a+1) i+a}+c(1, k) \theta\left(C k^{1+a}\right)\left(1-\frac{k+2 a}{(a+1) i+a}\right) \\
& +c(1, k-1) i \frac{k-1+a}{(a+1) i+a}++c(1, k-1) \theta\left(C(k-1)^{1+a}\right) \frac{k-1+a}{(a+1) i+a} \\
& +c(k) i \frac{k-1+a}{(a+1) i+a}+c(k) \theta_{1}\left(C_{1} k^{a+1}\right) \frac{k-1+a}{(a+1) i+a} \\
= & c(1, k)(i+1)+c(1, k) \theta\left(C k^{1+a}\right)\left(1-\frac{k+2 a}{(a+1) i+a}\right) \\
& -\frac{(k-1+a) a c(1, k-1)}{((a+1) i+a)(k+3 a+1)}-\frac{(k-1+a) a c(k)}{((a+1) i+a)(k+3 a+1)} \\
& +c(1, k-1) \theta\left(C(k-1)^{1+a}\right) \frac{k-1+a}{(a+1) i+a}+c(k) \theta_{1}\left(C_{1} k^{a+1}\right) \frac{k-1+a}{(a+1) i+a}
\end{aligned} .
$$

We want to prove that there exists a constant $C$ such that

$$
\begin{aligned}
& c(1, k) C k^{1+a} \frac{k+2 a}{(a+1) i+a} \\
& \geq \frac{(k-1+a) a c(1, k-1)}{((a+1) i+a)(k+3 a+1)}+\frac{(k-1+a) a c(k)}{((a+1) i+a)(k+3 a+1)} \\
& \quad+c(1, k-1) C(k-1)^{1+a} \frac{k-1+a}{(a+1) i+a}+c(k) C_{1} k^{1+a} \frac{k-1+a}{(a+1) i+a} .
\end{aligned}
$$

It is sufficient to prove that the following inequalities hold:

$$
\begin{aligned}
& \frac{c(1, k-1) C k^{1+a}(k+2 a)(k-1+a)}{((a+1) i+a)(k+3 a+1)} \\
& \quad \geq \frac{(k-1+a) a c(1, k-1)}{((a+1) i+a)(k+3 a+1)}+\frac{c(1, k-1) C(k-1)^{1+a}(k-1+a)}{(a+1) i+a}
\end{aligned}
$$


and

$$
\begin{aligned}
& \frac{c(k) C k^{1+a}(k+2 a)(k-1+a)}{((a+1) i+a)(k+3 a+1)} \\
& \quad \geq \frac{(k-1+a) a c(k)}{((a+1) i+a)(k+3 a+1)}+\frac{c(k) C_{1} k^{1+a}(k-1+a)}{(a+1) i+a},
\end{aligned}
$$

or

$$
\begin{aligned}
& C k^{1+a}(k+2 a) \geq a+C(k-1)^{1+a}(k+3 a+1), \\
& C k^{1+a}(k+2 a) \geq a+C_{1} k^{1+a}(k+3 a+1) .
\end{aligned}
$$

Note that

$$
\begin{aligned}
k^{1+a} & (k+2 a)-(k-1)^{1+a}(k+3 a+1) \\
= & k^{1+a}(k+2 a)-\left(k^{1+a}-(1+a) k^{a}+\frac{a(a+1)}{2} k^{a-1}\right. \\
& \left.\quad+O\left(k^{a-2}\right)\right)(k+3 a+1) \\
= & \frac{(5 a+2)(a+1)}{2} k^{a}+O\left(k^{a-1}\right) .
\end{aligned}
$$

For large values of $k$, there exists a constant $C$ such that

$$
C\left(k^{1+a}(k+2 a)-(k-1)^{1+a}(k+3 a+1)\right) \geq a .
$$

But we cannot choose a constant $C$ if

$$
k^{1+a}(k+2 a) \leq(k-1)^{1+a}(k+3 a+1) .
$$

There is a finite number of $k$ with

$$
\frac{(5 a+2)(a+1)}{2} k^{a}+O\left(k^{a-1}\right) \leq 0 .
$$

For such $k$, we want to prove that $\mathrm{E} N_{n}(1, k)=c(1, k)(n+O(f(k)))$ with some function $f(k)$. Using the method above, we obtain the same inequalities:

$$
\begin{aligned}
& f(k)(k+2 a) \geq a+f(k-1)(k+3 a+1), \\
& f(k)(k+2 a) \geq a+C_{1} k^{1+a}(k+3 a+1) .
\end{aligned}
$$

There exists a function $f$ such that the inequalities hold. This completes the proof for $\mathrm{E} N_{n}(1, k)$.

Consider the case $l>1$. Assume that we have already proved that

$$
\mathrm{E} N_{n}(i, j)=c(i, j)\left(n+\theta\left(C(i+j)^{1+a}\right)\right)
$$

for all $i$ and $j$ such that $i<l, j \leq k$, or $i \leq l, j<k$, and for all $n$. 
We use the following equality, which is similar to (4.2):

$$
\begin{aligned}
\mathrm{E} N_{i+1}(l, k)= & \mathrm{E}_{i}(l, k)\left(1-\frac{l(1+a)+k+a-1}{(a+1) i+a}\right) \\
& +\frac{(l-2+a) \mathrm{E} N_{i}(l-1, k)}{(a+1) i+a}+\frac{(k+a l-1) \mathrm{E} N_{i}(l, k-1)}{(a+1) i+a} .
\end{aligned}
$$

Note that if we have at least one vertex with first degree $l$ and second degree $k$ in $H_{a, 1}^{i}$ (without a loop), then we have at least $l+k-1$ edges in this graph. Therefore, $\mathrm{E} N_{i}(l, k)=0$ when $i<l+k-1$. Consider the case $i=l+k-1$. It is sufficient to prove that

$$
\mathrm{E} N_{l+k-1}(l, k) \leq C c(l, k)(l+k)
$$

with some $C$. For any finite number of small $l$ and $k$, we can easily find a constant $C$ such that

$$
\mathrm{E} N_{l+k-1}(l, k) \leq C c(l, k)(l+k)
$$

Using (4.3), we get

$$
\begin{aligned}
& \mathrm{E} N_{l+k-1}(l, k)=\frac{(l-2+a) \mathrm{E} N_{l+k-2}(l-1, k)}{(a+1)(l+k-2)+a}+\frac{(k+a l-1) \mathrm{E} N_{l+k-2}(l, k-1)}{(a+1)(l+k-2)+a} \\
& \quad \leq C c(l-1, k) \frac{(l-2+a)(l+k-1)}{(a+1)(l+k-2)+a}+C c(l, k-1) \frac{(k+a l-1)(l+k-1)}{(a+1)(l+k-2)+a} \\
& \quad \leq C c(l-1, k) \frac{(l-2+a)(l+k)}{l+a l+k+2 a}+C c(l, k-1) \frac{(a l+k-1)(l+k)}{l+a l+k+2 a} .
\end{aligned}
$$

The last inequality holds if $k$ is large enough.

We also need to consider a finite number of small $k$. First we show that for any finite number of small $k$ we have

$$
c(l, k)=\Omega\left(\frac{l^{k-4+\frac{a^{2}}{a+1}}}{(1+a)^{l}}\right) .
$$

Indeed, from the recurrence relation we obtain

$$
c(l, 1)=c(l-1,1) \frac{l-2+a}{(a+1)(l+1+a /(a+1))} .
$$

Therefore,

$$
c(l, 1)=\Omega\left(\frac{\Gamma(l-1+a)}{(a+1)^{l} \Gamma(l+2+a /(a+1))}\right)=\Omega\left(\frac{l^{-3+\frac{a^{2}}{a+1}}}{(1+a)^{l}}\right) .
$$

Here we used Lemma 4.1 from Section 4.2. For $k \geq 2$, we have

$$
c(l, k)=c(l, k-1) \frac{a l+k-1}{l(1+a)+k+2 a}+c(l-1, k) \frac{l-2+a}{l(1+a)+k+2 a} .
$$


It is sufficient to prove that there exists a positive function $f(k)$ such that

$$
\begin{aligned}
& f(k)(l(1+a)+k+2 a) l^{k-4+\frac{a^{2}}{a+1}} \\
& \quad \leq f(k-1)(a l+k-1) l^{k-5+\frac{a^{2}}{a+1}}+f(k)(l-2+a)(a+1)(l-1)^{k-4+\frac{a^{2}}{a+1}}
\end{aligned}
$$

and

$$
\begin{aligned}
& f(k)(l(1+a)+k+2 a)\left(l^{k-4+\frac{a^{2}}{a+1}}-(l-1)^{k-4+\frac{a^{2}}{a+1}}\right) \\
& \quad+f(k)\left(3 a+k-a^{2}+2\right)(l-1)^{k-4+\frac{a^{2}}{a+1}} \\
& \leq f(k-1)(a l+k-1) l^{k-5+\frac{a^{2}}{a+1}} .
\end{aligned}
$$

The last inequality holds for some positive function $f(k)$. We can choose any function $f(k)$ here, because we consider only a finite number of small $k$.

So we want to prove that

$$
\mathrm{E} N_{l+k-1}(l, k)=O\left(\frac{l^{k-3+\frac{a^{2}}{a+1}}}{(1+a)^{l}}\right) .
$$

Suppose that we have a graph on $l+k-1$ vertices and that a vertex $t$ has first degree $l$ and second degree $k$. Then one edge from this vertex goes to the vertex $1, l-1$ vertices send edges to $t$, and $k-2$ vertices send edges to the neighbors of $t$. There are $\left(\begin{array}{c}l+k-2 \\ k-2\end{array}\right)$ ways to choose our vertex $t$ and its neighbors. In each case, the probability that these neighbors will send edges to the vertex $t$ is

$$
\begin{aligned}
O\left(\frac{(a(a+1) \cdots(a+l-2))}{(3 a+2)(4 a+3) \cdots(l(a+1)+a)}\right) & =O\left(\frac{\Gamma(a+l-1)}{(a+1)^{l} \Gamma(l+1+a /(a+1))}\right) \\
& =O\left(\frac{l^{-2+\frac{a^{2}}{a+1}}}{(a+1)^{l}}\right),
\end{aligned}
$$

so

$$
\mathrm{E} N_{l+k-1}(l, k)=O\left(\frac{l^{k-4+\frac{a^{2}}{a+1}}}{(a+1)^{l}}\right) .
$$

This concludes the case $i=l+k-1$.

For $i \geq l+k-1$, we have

$$
\begin{aligned}
& \mathrm{E} N_{i+1}(l, k)=\mathrm{E} N_{i}(l, k)\left(1-\frac{l(1+a)+k+a-1}{(a+1) i+a}\right)+\frac{(l-2+a) \mathrm{E} N_{i}(l-1, k)}{(a+1) i+a} \\
& +\frac{(k+a l-1) \mathrm{E} N_{i}(l, k-1)}{(a+1) i+a}
\end{aligned}
$$




$$
\begin{aligned}
& =c(l, k)\left(i+\theta\left(C(l+k)^{1+a}\right)\right)\left(1-\frac{l(1+a)+k+a-1}{(a+1) i+a}\right) \\
& +c(l-1, k)\left(i+\theta\left(C(l+k-1)^{1+a}\right)\right) \frac{(l-2+a)}{(a+1) i+a} \\
& +c(l, k-1)\left(i+\theta\left(C(l+k-1)^{1+a}\right)\right) \frac{(k+a l-1)}{(a+1) i+a} \\
& =c(l, k) i-c(l, k) i \frac{l(1+a)+k+a-1}{(a+1) i+a} \\
& +c(l, k) \theta\left(C(l+k)^{1+a}\right)\left(1-\frac{l(1+a)+k+a-1}{(a+1) i+a}\right) \\
& +c(l-1, k) i \frac{(l-2+a)}{(a+1) i+a}+c(l, k-1) i \frac{(k+a l-1)}{(a+1) i+a} \\
& +c(l-1, k) \theta\left(C(l+k-1)^{1+a}\right) \frac{(l-2+a)}{(a+1) i+a} \\
& +c(l, k-1) \theta\left(C(l+k-1)^{1+a}\right) \frac{(k+a l-1)}{(a+1) i+a} \\
& =c(l, k)(i+1)-c(l, k) \frac{i l(1+a)+i k+2 i a+a}{(a+1) i+a}+c(l-1, k) i \frac{(l-2+a)}{(a+1) i+a} \\
& +c(l, k-1) i \frac{(k+a l-1)}{(a+1) i+a} \\
& +c(l, k) \theta\left(C(l+k)^{1+a}\right)\left(1-\frac{l(1+a)+k+a-1}{(a+1) i+a}\right) \\
& +c(l-1, k) \theta\left(C(l+k-1)^{1+a}\right) \frac{(l-2+a)}{(a+1) i+a} \\
& +c(l, k-1) \theta\left(C(l+k-1)^{1+a}\right) \frac{(k+a l-1)}{(a+1) i+a} \\
& =c(l, k)(i+1)-\frac{a(k+a l-1) c(l, k-1)}{((a+1) i+a)(l(1+a)+k+2 a)} \\
& -\frac{a(l-2+a) c(l-1, k)}{((a+1) i+a)(l(1+a)+k+2 a)} \\
& +c(l, k) \theta\left(C(l+k)^{1+a}\right)\left(1-\frac{l(1+a)+k+a-1}{(a+1) i+a}\right) \\
& +c(l-1, k) \theta\left(C(l+k-1)^{1+a}\right) \frac{(l-2+a)}{(a+1) i+a} \\
& +c(l, k-1) \theta\left(C(l+k-1)^{1+a}\right) \frac{(k+a l-1)}{(a+1) i+a} .
\end{aligned}
$$

We want the error term to be not greater than $C c(l, k)(l+k)^{1+a}$, and therefore, 
it is sufficient to prove the following inequality:

$$
\begin{aligned}
& C c(l, k)\left((l+k)^{1+a}\right) \frac{l(1+a)+k+a-1}{(a+1) i+a} \\
& \geq \frac{a(k+a l-1) c(l, k-1)}{((a+1) i+a)(l(1+a)+k+2 a)}+\frac{a(l-2+a) c(l-1, k)}{((a+1) i+a)(l(1+a)+k+2 a)} \\
& \quad+C c(l-1, k)(l+k-1)^{1+a} \frac{(l-2+a)}{(a+1) i+a} \\
& \quad+C c(l, k-1)(l+k-1)^{1+a} \frac{(k+a l-1)}{(a+1) i+a} .
\end{aligned}
$$

It is sufficient to show that the following inequalities hold:

$$
\begin{gathered}
C c(l, k-1)(l+k)^{1+a} \frac{(l(1+a)+k+a-1)(k+a l-1)}{((a+1) i+a)(l(1+a)+k+2 a)} \\
\geq \frac{a(k+a l-1) c(l, k-1)}{((a+1) i+a)(l(1+a)+k+2 a)} \\
\quad+C c(l, k-1)(l+k-1)^{1+a} \frac{(k+a l-1)}{(a+1) i+a}
\end{gathered}
$$

and

$$
\begin{aligned}
C c(l-1, k)(l+k)^{1+a} & \frac{(l(1+a)+k+a-1)(l-2+a)}{((a+1) i+a)(l(1+a)+k+2 a)} \\
\geq & \frac{a(l-2+a) c(l-1, k)}{((a+1) i+a)(l(1+a)+k+2 a)} \\
& +C c(l-1, k)(l+k-1)^{1+a} \frac{(l-2+a)}{(a+1) i+a} .
\end{aligned}
$$

In other words,

$$
\begin{aligned}
& C(l+k)^{1+a}(l(1+a)+k+a-1)(k+a l-1) \\
& \quad \geq a(k+a l-1)+C(l+k-1)^{1+a}(k+a l-1)(l(1+a)+k+2 a)
\end{aligned}
$$

and

$$
\begin{aligned}
& C(l+k)^{1+a}(l(1+a)+k+a-1)(l-2+a) \\
& \quad \geq a(l-2+a)+C(l+k-1)^{1+a}(l-2+a)(l(1+a)+k+2 a) .
\end{aligned}
$$

To prove both inequalities we make the following transformations:

$$
\begin{aligned}
& (l+k)^{1+a}(l(1+a)+k+a-1)-(l+k-1)^{1+a}(l(1+a)+k+2 a) \\
& \quad=(l+k)^{1+a}(l(1+a)+k+a-1)-\left((l+k)^{1+a}-(1+a)(l+k)^{a}\right.
\end{aligned}
$$




$$
\begin{aligned}
& \left.+\frac{a(1+a)}{2}(l+k)^{a-1}+O\left((l+k)^{a-2}\right)\right)(l(1+a)+k+2 a) \\
= & -(l+k)^{1+a}(1+a)+(1+a)(l+k)^{a}(l(1+a)+k+2 a) \\
& -\frac{a(1+a)}{2}(l+k)^{a-1}(l(1+a)+k+2 a) \\
& +O\left((l+k)^{a-2}\right)(l(1+a)+k+2 a) \\
= & (l+k)^{a-1}(1+a)\left(a l^{2}+a l k+2 a l+2 a k-\frac{a(1+a)}{2} l-\frac{a}{2} k-\frac{2 a^{2}}{2}\right) \\
& +O\left((l+k)^{a-2}\right)(l(1+a)+k+2 a) \\
= & (l+k)^{a-1}(1+a)\left(a l^{2}+a l k+\frac{3}{2} a l+\frac{3}{2} a k-\frac{1}{2} a^{2} l-a^{2}\right) \\
& +O\left((l+k)^{a-2}\right)(l(1+a)+k+2 a) .
\end{aligned}
$$

If $l$ or $k$ is large enough, then there exists a constant $C$ such that

$$
\begin{aligned}
& C(l+k)^{a-1}(1+a)\left(a l^{2}+a l k+\frac{3}{2} a l+\frac{3}{2} a k-\frac{1}{2} a^{2} l-a^{2}\right) \\
& \quad+O\left((l+k)^{a-2}\right)(l(1+a)+k+2 a) \geq a .
\end{aligned}
$$

Finally, we need to consider the finite number of small $l$ and $k$. We want to find some function $f(l, k)$ such that

$$
\begin{aligned}
& f(l, k) c(l, k)\left((l+k)^{1+a}\right) \frac{l(1+a)+k+a-1}{(a+1) i+a} \\
& \geq \frac{a(k+a l-1) c(l, k-1)}{((a+1) i+a)(l(1+a)+k+2 a)}+\frac{a(l-2+a) c(l-1, k)}{((a+1) i+a)(l(1+a)+k+2 a)} \\
& \quad+f(l-1, k) c(l-1, k) \frac{(l-2+a)}{(a+1) i+a}+f(l, k-1) c(l, k-1) \frac{(k+a l-1)}{(a+1) i+a} .
\end{aligned}
$$

Such a function $f(l, k)$ exists, because we can choose $f(l, k)$ growing in $l$ and $k$ as fast as we need. This concludes the proof of Theorem 2.5.

\subsection{Proof of Theorem 2.1}

In this proof we shall use the following lemma.

Lemma 4.I. For $t>0$ and fixed $a>0$,

$$
\frac{\Gamma(t+a)}{\Gamma(t)}=t^{a}(1+O(1 / t)) .
$$


Proof. From Stirling's formula we obtain

$$
\frac{\Gamma(t+a)}{\Gamma(t)}=\sqrt{\frac{t}{t+a}} \frac{(t+a)^{a}}{e^{a}}\left(\frac{t+a}{t}\right)^{t}(1+O(1 / t)) .
$$

It is easy to check that

$$
t \ln \left(1+\frac{a}{t}\right)=a+O(1 / t)
$$

So

$$
\left(1+\frac{a}{t}\right)^{t}=e^{a}(1+O(1 / t))
$$

We obtain

$$
\frac{\Gamma(t+a)}{\Gamma(t)}=\sqrt{\frac{t}{t+a}}(t+a)^{a}(1+O(1 / t))=t^{a}(1+O(1 / t)) .
$$

4.2.I. Estimation of $c(l, k)$. We begin with a lemma.

Lemma 4.2.

$$
c(1, k)=\frac{\Gamma(2 a+1)(1+O(1 / k))}{\Gamma(a) k^{a+1}} .
$$

Proof. As we know,

$$
c(k)=\frac{\mathrm{B}(k-1+a, a+2)}{\mathrm{B}(a, a+1)}=\frac{(a+1) \Gamma(2 a+1) \Gamma(k-1+a)}{\Gamma(a) \Gamma(k+1+2 a)} .
$$

Using the recurrence relation

$$
c(1, k)=c(1, k-1) \frac{a+k-1}{k+3 a+1}+c(k) \frac{a+k-1}{k+3 a+1},
$$

we obtain

$$
\begin{aligned}
c(1, k) & =\sum_{j=1}^{k} \frac{c(j)(a+j-1) \cdots(a+k-1)}{(j+3 a+1) \cdots(k+3 a+1)} \\
& =\frac{(a+1) \Gamma(2 a+1)}{\Gamma(a)} \sum_{j=1}^{k} \frac{\Gamma(j-1+a)(a+j-1) \cdots(a+k-1)}{\Gamma(j+1+2 a)(j+3 a+1) \cdots(k+3 a+1)} \\
& =\frac{(a+1) \Gamma(2 a+1) \Gamma(a+k)}{\Gamma(a) \Gamma(k+3 a+2)} \sum_{j=1}^{k} \frac{\Gamma(j+3 a+1)}{\Gamma(j+1+2 a)}
\end{aligned}
$$




$$
\begin{aligned}
& =\frac{(a+1) \Gamma(2 a+1) \Gamma(a+k)}{\Gamma(a) \Gamma(k+3 a+2)} \sum_{j=1}^{k} j^{a}(1+O(1 / j)) \\
& =\frac{\Gamma(2 a+1) k^{a+1}(1+O(1 / k))}{\Gamma(a) k^{2 a+2}}=\frac{\Gamma(2 a+1)(1+O(1 / k))}{\Gamma(a) k^{a+1}} .
\end{aligned}
$$

4.2.2. Sum of $c(l, k)$. We want to estimate the sum $\sum_{l=1}^{\infty} c(l, k)$. First let us prove that the series $\sum_{l=1}^{\infty} l^{N} c(l, k)$ converges for all $N$ and $k$.

The inequality

$$
c(l, k) \leq \tilde{C} \frac{p^{k}}{(1+q)^{l}}
$$

holds for every $p>1$ and $q=\min \{a, 1\} \frac{(p-1)}{p}$. Here we choose $\tilde{C}$ such that $\tilde{C} \frac{p^{k}}{1+a p} \geq c(1, k)$ for every $k$. We need to prove that

$$
\frac{p^{k}}{(1+q)^{l}}(l+a l+k+2 a) \geq \frac{p^{k-1}}{(1+q)^{l}}(a l+k-1)+\frac{p^{k}}{(1+q)^{l-1}}(l-2+a) .
$$

We perform some transformations:

$$
\begin{aligned}
p(l+a l+k+2 a) & \geq(a l+k-1)+p(1+q)(l-2+a), \\
p(a l+k+a+2) & \geq(a l+k-1)+p q(l-2+a), \\
a l+k+a+2 & \geq \min \{a, 1\}(l-2+a) .
\end{aligned}
$$

The last inequality holds. Therefore, $c(l, k) \leq \tilde{C}_{\frac{p^{k}}{(1+q)^{l}}}$, and $\sum_{l=1}^{\infty} l^{N} c(l, k)$ converges.

For $l \geq 2$ and every $c \geq 0$ we have

$$
\begin{aligned}
& c(l, k)(l(1+a)+k+2 a) \frac{\Gamma(l+a+c)}{\Gamma(l+a-1)} \\
& \quad=c(l, k-1) \frac{\Gamma(l+a+c)}{\Gamma(l+a-1)}(a l+k-1)+c(l-1, k)(l-2+a) \frac{\Gamma(l+a+c)}{\Gamma(l+a-1)} .
\end{aligned}
$$

Therefore,

$$
\begin{aligned}
& \sum_{l=2}^{\infty} c(l, k)(l(1+a)+k+2 a) \frac{\Gamma(l+a+c)}{\Gamma(l+a-1)} \\
& \quad=\sum_{l=2}^{\infty} c(l, k-1)(a l+k-1) \frac{\Gamma(l+a+c)}{\Gamma(l+a-1)}+\sum_{l=1}^{\infty} c(l, k) \frac{\Gamma(l+a+c+1)}{\Gamma(l+a-1)}
\end{aligned}
$$


and

$$
\begin{aligned}
& \sum_{l=2}^{\infty} c(l, k)(a l+k+a-c) \frac{\Gamma(l+a+c)}{\Gamma(l+a-1)} \\
& \quad=\sum_{l=2}^{\infty} c(l, k-1)(a l+k-1) \frac{\Gamma(l+a+c)}{\Gamma(l+a-1)}+c(1, k) \frac{\Gamma(a+c+2)}{\Gamma(a)}
\end{aligned}
$$

Consider the function

$$
f_{c}(k)=\frac{\Gamma(k+a-c)}{\Gamma(k)}=k^{a-c}(1+O(1 / k)) .
$$

It is easy to see that

$$
\frac{f_{c}(k+1)}{f_{c}(k)}=1+\frac{a-c}{k} .
$$

We have

$$
\begin{aligned}
& \sum_{j=1}^{k} \sum_{l=2}^{\infty} c(l, j)(a l+j+a-c) \frac{\Gamma(l+a+c)}{\Gamma(l+a-1)} f_{c}(j) \\
& =\sum_{j=1}^{k} \sum_{l=2}^{\infty} c(l, j-1)(a l+j-1) \frac{\Gamma(l+a+c)}{\Gamma(l+a-1)} f_{c}(j) \\
& \quad+\sum_{j=1}^{k} c(1, j) \frac{\Gamma(a+c+2)}{\Gamma(a)} f_{c}(j), \\
& \sum_{j=1}^{k} \sum_{l=2}^{\infty} c(l, j)(a l+j+a-c) \frac{\Gamma(l+a+c)}{\Gamma(l+a-1)} f_{c}(j) \\
& =\sum_{j=1}^{k-1} \sum_{l=2}^{\infty} c(l, j)(a l+j) \frac{\Gamma(l+a+c)}{\Gamma(l+a-1)} f_{c}(j)\left(1+\frac{a-c}{j}\right) \\
& \quad+\sum_{j=1}^{k} c(1, j) \frac{\Gamma(a+c+2)}{\Gamma(a)} f_{c}(j), \\
& \sum_{l=2}^{\infty} c(l, k)(a l+k+a-c) \frac{\Gamma(l+a+c)}{\Gamma(l+a-1)} f_{c}(k) \\
& \quad=\sum_{j=1}^{k-1} \sum_{l=2}^{\infty} c(l, j) \frac{\Gamma(l+a+c)}{\Gamma(l+a-1)} \frac{a l(a-c)}{j} f_{c}(j)+\sum_{j=1}^{k} c(1, j) \frac{\Gamma(a+c+2)}{\Gamma(a)} f_{c}(j) .
\end{aligned}
$$


If $c \geq a$, then taking into account Lemma 4.2 and the above-mentioned asymptotics for $f_{c}(j)$, we have

$$
\begin{aligned}
\sum_{l=2}^{\infty} c(l, k)(a l+k+a-c) \frac{\Gamma(l+a+c)}{\Gamma(l+a-1)} f_{c}(k) & \leq \sum_{j=1}^{k} c(1, j) \frac{\Gamma(a+c+2)}{\Gamma(a)} f_{c}(j) \\
& =O(1)
\end{aligned}
$$

Hence,

$$
\sum_{l=2}^{\infty} c(l, k) \frac{\Gamma(l+a+c)}{\Gamma(l+a-1)} f_{c}(k)=O(1 / k) .
$$

We want to prove that for every $0 \leq c<a+1$, the following equality holds:

$$
\sum_{l=2}^{\infty} c(l, k) \frac{\Gamma(l+a+c)}{\Gamma(l+a-1)} f_{c}(k)=O\left(\frac{(\ln k)^{\lceil a-c\rceil}}{k}\right) .
$$

We have already proved this statement for $a \leq c<a+1$.

Suppose that for $c^{\prime} \geq 1$ we have

$$
\sum_{l=2}^{\infty} c(l, k) \frac{\Gamma\left(l+a+c^{\prime}\right)}{\Gamma(l+a-1)} f_{c^{\prime}}(k)=O\left(\frac{(\ln k)^{\left\lceil a-c^{\prime}\right\rceil}}{k}\right) .
$$

Then

$$
\begin{aligned}
\sum_{l=2}^{\infty} c & (l, k)\left(a l+k+a-c^{\prime}+1\right) \frac{\Gamma\left(l+a+c^{\prime}-1\right)}{\Gamma(l+a-1)} f_{c^{\prime}-1}(k) \\
= & \sum_{j=1}^{k-1} \sum_{l=2}^{\infty} c(l, j) \frac{\Gamma\left(l+a+c^{\prime}-1\right)}{\Gamma(l+a-1)} \frac{a l\left(a-c^{\prime}+1\right)}{j}\left(j+a-c^{\prime}\right) f_{c^{\prime}}(j) \\
& +\sum_{j=1}^{k} c(1, j) \frac{\Gamma\left(a+c^{\prime}+1\right)}{\Gamma(a)} f_{c^{\prime}-1}(j) \\
= & O\left(\sum_{j=1}^{k-1} \frac{(\ln k)^{\left\lceil a-c^{\prime}\right\rceil}}{j}\right) \\
= & O\left((\ln k)^{\left\lceil a-c^{\prime}+1\right\rceil}\right)
\end{aligned}
$$

We have proved (4.4). In particular,

$$
\sum_{l=2}^{\infty} c(l, k) \frac{\Gamma(l+a)}{\Gamma(l+a-1)} f_{0}(k)=\sum_{l=2}^{\infty} c(l, k)(l+a-1) f_{0}(k)=O\left(\frac{(\ln k)^{\lceil a\rceil}}{k}\right) .
$$


Put $x_{k}=\sum_{l=2}^{\infty} c(l, k)$. For $l \geq 2$,

$$
c(l, k)(l(1+a)+k+2 a)=c(l, k-1)(a l+k-1)+c(l-1, k)(l-2+a) .
$$

So

$$
\begin{aligned}
\sum_{l=2}^{\infty} c(l, k)(l(1+a)+k+2 a) & =\sum_{l=2}^{\infty} c(l, k-1)(a l+k-1)+\sum_{l=1}^{\infty} c(l, k)(l-1+a), \\
\sum_{l=2}^{\infty} c(l, k)(a l+k+a+1) & =\sum_{l=2}^{\infty} c(l, k-1)(a l+k-1)+a c(1, k),
\end{aligned}
$$

and

$$
(k+a+1) x_{k}=(k-1) x_{k-1}+a c(1, k)+a \sum_{l=2}^{\infty} l(c(l, k-1)-c(l, k)) .
$$

We have

$$
\begin{aligned}
(k+a+1) f_{-1}(k) x_{k}= & (k-1) f_{-1}(k) x_{k-1}+a f_{-1}(k) c(1, k) \\
& +a f_{-1}(k) \sum_{l=2}^{\infty} l(c(l, k-1)-c(l, k)), \\
\sum_{j=1}^{k}(j+a+1) f_{-1}(j) x_{j}= & \sum_{j=1}^{k-1} f_{-1}(j)(j+a+1) x_{j}+\sum_{j=1}^{k} a f_{-1}(j) c(1, j) \\
& +\sum_{j=1}^{k} a f_{-1}(j) \sum_{l=2}^{\infty} l(c(l, j-1)-c(l, j)), \\
(k+a+1) f_{-1}(k) x_{k}= & a \sum_{j=1}^{k} f_{-1}(j) c(1, j) \\
& +a \sum_{j=1}^{k} f_{-1}(j) \sum_{l=2}^{\infty} l(c(l, j-1)-c(l, j)),
\end{aligned}
$$

and

$$
\begin{aligned}
f_{-1}(k)(k+a+1) x_{k}= & a \sum_{j=1}^{k} f_{-1}(j) c(1, j)+a(a+1) \sum_{j=1}^{k-1} \frac{f_{-1}(j)}{j} \sum_{l=2}^{\infty} l c(l, j) \\
& -a f_{-1}(k) \sum_{l=2}^{\infty} l c(l, k) \\
= & a \sum_{j=1}^{k} j^{a+1} \frac{\Gamma(2 a+1)}{\Gamma(a) j^{a+1}}(1+O(1 / j))+\sum_{j=1}^{k-1} O\left(\frac{(\ln j)^{\lceil a\rceil}}{j}\right)
\end{aligned}
$$




$$
\begin{aligned}
& +O\left((\ln k)^{\lceil a\rceil}\right) \\
= & a k \frac{\Gamma(2 a+1)}{\Gamma(a)}+\sum_{j=1}^{k-1} O\left(\frac{(\ln j)^{\lceil a\rceil}}{j}\right)+O\left((\ln k)^{\lceil a\rceil}\right) \\
= & a k \frac{\Gamma(2 a+1)}{\Gamma(a)}\left(1+O\left(\frac{(\ln k)^{\lceil a+1\rceil}}{k}\right)\right) .
\end{aligned}
$$

Here we used (4.5) and Lemma 4.2. We obtain

$$
x_{k}=\frac{a \Gamma(2 a+1)}{\Gamma(a) k^{a+1}}\left(1+O\left(\frac{(\ln k)^{\lceil a+1\rceil}}{k}\right)\right)
$$

and

$$
\sum_{l=1}^{\infty} c(l, k)=c(1, k)+x_{k}=\frac{(a+1) \Gamma(2 a+1)}{\Gamma(a) k^{a+1}}\left(1+O\left(\frac{(\ln k)^{\lceil a+1\rceil}}{k}\right)\right) .
$$

4.2.3. Estimation of $E Y_{n}(k)$. Note that

$$
\begin{aligned}
\sum_{l \geq 1} \sum_{j \geq k} \mathrm{EN}_{i+1}(l, j)= & \sum_{l \geq 1} \sum_{j \geq k} \mathrm{E} N_{i}(l, j)+\sum_{l \geq 1} \frac{(a l+k-1) \mathrm{E} N_{i}(l, k-1)}{(a+1) i+a} \\
& +\sum_{j \geq k} \frac{(j-1+a) M_{i}^{1}(j)}{(a+1) i+a} .
\end{aligned}
$$

Therefore we obtain

$$
\begin{aligned}
\sum_{l \geq 1} \sum_{j \geq k} \mathrm{E} N_{n}(l, j)= & \sum_{i=1}^{n-1} \sum_{l \geq 1} \frac{(a l+k-1) \mathrm{E} N_{i}(l, k-1)}{(a+1) i+a} \\
& +\sum_{i=1}^{n-1} \sum_{j \geq k} \frac{(j-1+a) M_{i}^{1}(j)}{(a+1) i+a} .
\end{aligned}
$$

Let us estimate the sum

$$
\sum_{i=1}^{n-1} \sum_{j \geq k} \frac{(j-1+a) M_{i}^{1}(j)}{(a+1) i+a}
$$

First we compute

$$
F_{t}(k)=\sum_{j \geq k}(j-1+a) M_{t}^{1}(j)
$$

Let us prove by induction on $k$ that

$$
F_{n}(k)=\frac{(a+1) \Gamma(2 a+1) \Gamma(k+a)}{\Gamma(a+1) \Gamma(k+2 a)} n\left(1+\theta\left(\frac{C(k-1)^{1+a}}{n}\right)\right)
$$


with some constant $C$. For $k=1$ and $k=2$, we have

$$
F_{n}(1)=\sum_{j \geq 1}(j-1+a) M_{n}^{1}(j)=n(1+a)
$$

and

$$
\begin{aligned}
F_{n}(2) & =F_{n}(1)-a M_{n}^{1}(1)=n(1+a)-a n \frac{(1+a)}{(2 a+1)}+O(1) \\
& =\frac{(1+a)^{2} n}{2 a+1}(1+O(1 / n)) .
\end{aligned}
$$

For $k \geq 3$ we have

$$
M_{i+1}^{1}(j)=M_{i}^{1}(j)\left(1-\frac{j-1+a}{(a+1) i+a}\right)+M_{i}^{1}(j-1) \frac{j-2+a}{(a+1) i+a} .
$$

We multiply this equality by $(j-1+a)$ and sum over all $j \geq k$ :

$$
\begin{aligned}
F_{i+1}(k)= & \sum_{j \geq k}(j-1+a) M_{i+1}^{1}(j) \\
= & \sum_{j \geq k}(j-1+a) M_{i}^{1}(j)-\sum_{j \geq k} M_{i}^{1}(j) \frac{(j-1+a)(j-1+a)}{(a+1) i+a} \\
& +\sum_{j \geq k-1} M_{i}^{1}(j) \frac{(j+a)(j-1+a)}{(a+1) i+a} \\
= & F_{i}(k)+\sum_{j \geq k} M_{i}^{1}(j) \frac{(j-1+a)}{(a+1) i+a}+M_{i}^{1}(k-1) \frac{(k-1+a)(k-2+a)}{(a+1) i+a} \\
= & F_{i}(k)\left(1+\frac{1}{(a+1) i+a}\right)+\left(F_{i}(k-1)-F_{i}(k)\right) \frac{(k-1+a)}{(a+1) i+a} \\
= & F_{i}(k)\left(1-\frac{k-2+a}{(a+1) i+a}\right)+F_{i}(k-1) \frac{(k-1+a)}{(a+1) i+a} .
\end{aligned}
$$

Note that for $i+1<k-1$ we have $F_{i+1}(k)=0$. Consider $i+1 \geq k-1$. Using the inductive hypothesis, we get

$$
\begin{aligned}
F_{i+1}(k) & \\
= & \frac{(a+1) \Gamma(2 a+1) \Gamma(k+a)}{\Gamma(a+1) \Gamma(k+2 a)} i\left(1-\frac{k-2+a}{(a+1) i+a}\right)\left(1+\theta\left(\frac{C(k-1)^{1+a}}{i}\right)\right) \\
& +\frac{(a+1) \Gamma(2 a+1) \Gamma(k-1+a) i}{\Gamma(a+1) \Gamma(k-1+2 a)} \frac{(k-1+a)}{(a+1) i+a}\left(1+\theta\left(\frac{C(k-2)^{1+a}}{i}\right)\right) \\
= & \frac{(a+1) \Gamma(2 a+1) \Gamma(k+a)}{\Gamma(a+1) \Gamma(k+2 a)}\left(i+1-\frac{a}{(a+1) i+a}\right.
\end{aligned}
$$




$$
\begin{aligned}
& +i\left(1-\frac{k-2+a}{(a+1) i+a}\right) \theta\left(\frac{C(k-1)^{1+a}}{i}\right) \\
& \left.+\frac{(k-1+2 a) i}{(a+1) i+a} \theta\left(\frac{C(k-2)^{1+a}}{i}\right)\right) .
\end{aligned}
$$

And we need to show that for some constant $C$,

$$
\frac{a}{(a+1) i+a}+\frac{(k-1+2 a)}{(a+1) i+a} C(k-2)^{1+a} \leq \frac{k-2+a}{(a+1) i+a} C(k-1)^{1+a} .
$$

This inequality holds for sufficiently large $C$.

We have

$$
\begin{aligned}
\sum_{i=1}^{n-1} \frac{F_{i}(k)}{(a+1) i+a} & =\sum_{i=1}^{n-1} \frac{\Gamma(2 a+1) \Gamma(k+a)}{\Gamma(a+1) \Gamma(k+2 a)}\left(1+O\left(\frac{k^{1+a}}{i}\right)\right) \\
& =\frac{\Gamma(2 a+1) \Gamma(k+a)}{\Gamma(a+1) \Gamma(k+2 a)} n\left(1+O\left(\frac{k^{1+a}}{n}\right)\right) .
\end{aligned}
$$

Let us estimate the sum

$$
\sum_{i=1}^{n-1} \sum_{l \geq 1} \frac{(a l+k-1) \mathrm{E} N_{i}(l, k-1)}{(a+1) i+a} .
$$

We start with the sum

$$
\sum_{l \geq 1}(a l+k-1) \mathrm{E} N_{i}(l, k-1)
$$

It is easy to see that

$$
\mathrm{E} N_{i}(l, k)=O(c(l, k) i)
$$

To verify this, one can follow the proof of Theorem 2.5 and make sure that it works for the inequality

$$
\mathrm{E} N_{i}(l, k)<\tilde{C} c(l, k)((a+1) i+a)
$$

with some constant $\tilde{C}$; note that the analogue of Lemma 2.6 is also needed.

Therefore,

$$
\sum_{l \geq 1}(a l-1) \mathrm{E} N_{i}(l, k-1)=O\left(\sum_{l \geq 1}(a l-1) c(l, k-1) i\right)=O\left(\frac{(\ln k)^{\lceil a\rceil} i}{k^{a+1}}\right) .
$$


Using (4.4), we obtain

$$
\begin{aligned}
\sum_{l \geq 1} k \mathrm{EN}_{i}(l, k-1) & =\sum_{l \geq 1} k c(l, k-1) i\left(1+O\left((l+k)^{1+a} / i\right)\right) \\
& =\frac{(a+1) \Gamma(2 a+1)}{\Gamma(a) k^{a}} i\left(1+O\left(\frac{(\ln k)^{\lceil a+1\rceil}}{k}\right)+O\left(\frac{k^{1+a}}{i}\right)\right) .
\end{aligned}
$$

Here we used the following estimate:

$$
\begin{aligned}
\sum_{l \geq 1} k c(l, k-1)(l+k)^{1+a} & =O\left(\sum_{l=1}^{k} k^{2+a} c(l, k-1)+\sum_{l \geq k} k c(l, k-1) l^{1+a}\right) \\
& =O(k)+O(1)=O(k) .
\end{aligned}
$$

So we have

$$
\begin{aligned}
& \sum_{i=1}^{n-1} \sum_{l \geq 1} \frac{(a l+k-1) \mathrm{E} N_{i}(l, k-1)}{(a+1) i+a} \\
& \quad=\frac{\Gamma(2 a+1)}{\Gamma(a) k^{a}} n\left(1+O\left(\frac{(\ln k)^{\lceil a+1\rceil}}{k}\right)+O\left(\frac{k^{1+a}}{n}\right)\right) .
\end{aligned}
$$

Hence

$$
\begin{aligned}
\sum_{l \geq 1} \sum_{j \geq k} \mathrm{E} N_{n}(l, j)= & \frac{a \Gamma(2 a+1)}{\Gamma(a+1) k^{a}} n\left(1+O\left(\frac{(\ln k)^{\lceil a+1\rceil}}{k}\right)\right) \\
& +\frac{\Gamma(2 a+1) \Gamma(k+a)}{\Gamma(a+1) \Gamma(k+2 a)} n\left(1+O\left(\frac{k^{1+a}}{n}\right)\right) \\
= & \frac{(a+1) \Gamma(2 a+1)}{\Gamma(a+1) k^{a}} n\left(1+O\left(\frac{(\ln k)^{\lceil a+1\rceil}}{k}\right)+O\left(\frac{k^{1+a}}{n}\right)\right) .
\end{aligned}
$$

Consider vertices with loops. For $k=0$, we have

$$
\sum_{l \geq 1} \sum_{j \geq 0} \mathrm{E} P_{n}(l, j)=\sum_{i=1}^{n} \frac{a}{(1+a) i-1}=O(\ln n)
$$

For $k \geq 2$, we have

$$
\sum_{l \geq 1} \sum_{j \geq k} \mathrm{E} P_{i+1}(l, j)=\sum_{l \geq 1} \sum_{j \geq k} \mathrm{E} P_{i}(l, j)+\sum_{l \geq 1} \frac{(a l+k-2 a-1) \mathrm{E} P_{i}(l, k-1)}{(a+1) i+a} .
$$


Therefore, we obtain

$$
\begin{aligned}
\sum_{l \geq 1} \sum_{j \geq k} \mathrm{E} P_{n}(l, j) & =\sum_{i=1}^{n-1} \sum_{l \geq 1} \frac{(a l+k-2 a-1) \mathrm{E} P_{i}(l, k-1)}{(a+1) i+a} \\
& \leq \sum_{i=1}^{n-1} \sum_{l \geq 1} \frac{(a l+k-2 a-1) p(l, k-1)}{(a+1) i} .
\end{aligned}
$$

From the recurrence relation for $p(l, k)$, it follows that

$$
p(l, k)=O\left(\frac{1}{l^{2}}\right) \quad \text { and } \quad p(l, k)=O\left(\frac{k}{l^{3}}\right) .
$$

To obtain the second estimate, consider $q(l, k)=p(l, k) / k$. For $k \geq 1$ we have

$$
\begin{aligned}
& q(l, k)(l+a l+k-1-a) \\
& \quad=q(l, k-1) \frac{(k-1)(a l+k-2 a-1)}{k}+q(l-1, k)(l-2+a)
\end{aligned}
$$

and

$$
\begin{aligned}
& q(l, k)(l+a l+k-1-a)-q(l, k-1)\left(a l+k-2 a-2-\frac{a l-2 a-1}{k}\right) \\
& \quad=q(l-1, k)(l-2+a) .
\end{aligned}
$$

Thus, $q(l, k)=O(q(l))$, where

$$
q(l)(l+a+1+(a l-2 a-1))=q(l-1)(l-2+a) .
$$

From this equality it follows that $q(l)=O\left(\frac{1}{l^{3}}\right)$.

We can estimate the following sum:

$$
\sum_{l \geq 1} \frac{(a l+k-2 a-1) p(l, k-1)}{(a+1)}=O(k) .
$$

Hence

$$
\sum_{l \geq 1} \sum_{j \geq k} \mathrm{E} P_{n}(l, j)=O(k \ln n) .
$$

Now we are ready to estimate $\mathrm{E} Y_{n}(k)$ :

$$
\begin{aligned}
\mathrm{E} Y_{n}(k) & =\sum_{l \geq 1} \sum_{j \geq k} \mathrm{E} N_{n}(l, j)+\sum_{l \geq 1} \sum_{j \geq k} \mathrm{E} P_{n}(l, k) \\
& =\frac{(a+1) \Gamma(2 a+1)}{\Gamma(a+1) k^{a}} n\left(1+O\left(\frac{(\ln k)^{\lceil a+1\rceil}}{k}\right)+O\left(\frac{k^{1+a}}{n}\right)\right)+O(k \ln n) \\
& =\frac{(a+1) \Gamma(2 a+1)}{\Gamma(a+1) k^{a}} n\left(1+O\left(\frac{(\ln k)^{\lceil a+1\rceil}}{k}\right)+O\left(\frac{k^{1+a}}{n}\right)\right)
\end{aligned}
$$


This concludes the proof of Theorem 2.1.

\subsection{Proof of Lemma 2.7}

It is easy to see that $\mathrm{E} P_{n}(0, k)=\mathrm{E} P_{n}(1, k)=0$. For all $k>0$, we have $\mathrm{E} P_{n}(2, k)=0$. For $k=0$, we have

$$
\begin{aligned}
\mathrm{E} P_{n}(2,0) & =\sum_{i=1}^{n} \frac{a}{(a+1) i-1} \prod_{j=i+1}^{n} \frac{(1+a) j-2-a}{(1+a) j-1} \\
& =\sum_{i=1}^{n} \frac{a}{(a+1) i-1} \frac{\Gamma\left(n-\frac{1}{a+1}\right) \Gamma\left(i+\frac{a}{a+1}\right)}{\Gamma\left(n+\frac{a}{a+1}\right) \Gamma\left(i-\frac{1}{a+1}\right)} \\
& =\frac{1}{n}(1+O(1 / n)) \sum_{i=1}^{n} \frac{a i}{(a+1) i-1}(1+O(1 / i))=O(1) .
\end{aligned}
$$

The rest of the proof is by induction. Consider $l \geq 3, k \geq 1$. Assume that we already proved that $\mathrm{E}_{n}(i, j) \leq p(i, j)$ for all $i$ and $j$ such that $i<l, j \leq k$ or $i \leq l, j<k$, and for all $n$. We use the following equality:

$$
\begin{aligned}
\mathrm{E} P_{i+1}(l, k)= & \mathrm{E} P_{i}(l, k)\left(1-\frac{l(a+1)+k-a-1}{(a+1) i+a}\right) \\
& +\mathrm{E} P_{i}(l, k-1) \frac{a l+k-2 a-1}{(a+1) i+a}+\mathrm{E} P_{i}(l-1, k) \frac{l-2+a}{(a+1) i+a} .
\end{aligned}
$$

Note that if we have at least one vertex with a loop, with first degree $l$ and second degree $k$ in the graph $H_{a, 1}^{i}$, then we have at least $l+k-1$ edges in the graph. Therefore, $\mathrm{E} P_{i}(l, k)=0$ if $i<l+k-1$. Consider the case $i=l+k-1$. Using (4.6), we get (for $k \geq 1$ )

$$
\begin{aligned}
\mathrm{E} P_{l+k-1}(l, k)= & \frac{(l-2+a) \mathrm{E} P_{l+k-2}(l-1, k)}{(a+1)(l+k-2)+a} \\
& +\frac{(a l+k-2 a-1) \mathrm{E} P_{l+k-2}(l, k-1)}{(a+1)(l+k-2)+a} \\
\leq & \frac{(l-2+a) p(l-1, k)}{(a+1)(l+k-2)+a}+\frac{(a l+k-2 a-1) p(l, k-1)}{(a+1)(l+k-2)+a} \\
= & \frac{(l+a l+k-1-a) p(l, k)}{(a+1)(l+k-2)+a} \leq p(l, k) .
\end{aligned}
$$

The last inequality holds for $k \geq 1 / a$. Consider the case $k<1 / a$. As in the proof of Theorem 2.5, we first estimate $p(l, k)$ :

$$
p(l, k)=\Omega\left(\frac{l^{k+\frac{a^{2}}{a+1}}}{(1+a)^{l}}\right) .
$$


For $k=0$, we have

$$
p(l, 0)=p(l-1,0) \frac{l-2+a}{(1+a)\left(l-1-\frac{1}{a+1}\right)} .
$$

Therefore,

$$
p(l, 0)=\Omega\left(\frac{l^{\frac{a^{2}}{a+1}}}{(1+a)^{l}}\right)
$$

For $k \geq 1$, we have

$$
p(l, k)=p(l, k-1) \frac{a l+k-2 a-1}{l(1+a)+k-1-a}+p(l-1, k) \frac{l-2+a}{l(1+a)+k-1-a} .
$$

Again, it suffices to prove that there exists a positive function $f(k)$ such that for large $l$,

$$
\begin{aligned}
& f(k)(l(1+a)+k-1-a) l^{k+\frac{a^{2}}{a+1}} \\
& \quad \leq f(k-1)(a l+k-2 a-1) l^{k+\frac{a^{2}}{a+1}-1}+f(k)(l-2+a)(a+1)(l-1)^{k+\frac{a^{2}}{a+1}}
\end{aligned}
$$

and

$$
\begin{aligned}
& f(k)(l(1+a)+k-1-a)\left(l^{k+\frac{a^{2}}{a+1}}-(l-1)^{k+\frac{a^{2}}{a+1}}\right) \\
& \quad+f(k)\left(k-a^{2}+1\right)(l-1)^{k+\frac{a^{2}}{a+1}} \\
& \leq f(k-1)(a l+k-2 a-1) l^{k+\frac{a^{2}}{a+1}-1} .
\end{aligned}
$$

The last inequality holds for some function $f(k)$.

We want to prove that

$$
\mathrm{E} P_{l+k-1}(l, k)=O\left(\frac{l^{k+\frac{a^{2}}{a+1}}}{(1+a)^{l}}\right)
$$

There are $l^{k}$ possible graphs on $l+k-1$ vertices with some vertex of first degree $l$, second degree $k$, and without a loop. And this vertex is exactly the vertex 1 . The probability for this vertex to be a vertex with first degree $l$ and second degree $k$ is

$$
O\left(\frac{l^{k}((a+1) \cdots(a+l-2))}{(a+2) \cdots((l-1)(a+1)-1)}\right)=O\left(\frac{l^{k+\frac{a^{2}}{a+1}}}{(a+1)^{l}}\right) .
$$

This concludes the case $i=l+k-1$. 
If $i \geq l+k-1$, then

$$
\begin{aligned}
\mathrm{E} P_{i+1}(l, k)= & \mathrm{E} P_{i}(l, k)\left(1-\frac{l(a+1)+k-a-1}{(a+1) i+a}\right) \\
& +\mathrm{E} P_{i}(l, k-1) \frac{a l+k-2 a-1}{(a+1) i+a}+\mathrm{E} P_{i}(l-1, k) \frac{l-2+a}{(a+1) i+a} .
\end{aligned}
$$

Using the recurrence relation for $p(l, k)$ and induction on $i$, it is easy to prove that $\mathrm{E} P_{n}(l, k) \leq p(l, k)$. This concludes the proof of Lemma 2.7.

\subsection{Proof of Theorem 2.3}

We estimate the expectation of $X_{n}(k)$ as follows:

$$
\begin{aligned}
\mathrm{E} X_{n}(k) & =\sum_{l=1}^{\infty} \mathrm{E} N_{n}(l, k)+\sum_{l=1}^{\infty} \mathrm{E} P_{n}(l, k) \\
& =\sum_{l=1}^{\infty} c(l, k) n+O\left(\sum_{l=1}^{\infty} c(l, k)(l+k)^{1+a}\right)+O\left(\sum_{l=1}^{\infty} p(l, k)\right) \\
& =\frac{(a+1) \Gamma(2 a+1) n}{\Gamma(a) k^{a+1}}\left(1+O\left(\frac{(\ln k)^{\lceil a+1\rceil}}{k}\right)\right)+O(1)+O(1) \\
& =\frac{(a+1) \Gamma(2 a+1) n}{\Gamma(a) k^{a+1}}\left(1+O\left(\frac{(\ln k)^{\lceil a+1\rceil}}{k}\right)+O\left(\frac{k^{1+a}}{n}\right)\right) .
\end{aligned}
$$

\section{References}

[Alon and Spencer 02] N. Alon and J. H. Spencer. The Probabilistic Method. WileyInterscience, 2002.

[Azuma 67] K. Azuma. "Weighted Sums of Certain Dependent Variables." Tôhoku Math. J. 19 (1967), 357-367.

[Barabási and Albert 99] A.-L. Barabási and R. Albert. "Emergence of Scaling in Random Networks." Science 286 (1999), 509-512.

[Berger et al. 09] N. Berger, C. Borgs, J. T. Chayes, and A. Saberi. "Weak Local Limits for Preferential Attachment Graphs." Preprint available from World Wide Web, 2009 (http://www.stanford.edu/class/msande337/notes/bslimit-old.pdf).

[Bollobás 01] B. Bollobás. Random Graphs, second edition. Cambridge Univ. Press, 2001.

[Bollobás and Riordan 03] B. Bollobás and O. M. Riordan. Mathematical Results on Scale-Free Random Graphs, Handbook of Graphs and Networks. Wiley-VCH, 2003.

[Bollobás and Riordan 04] B. Bollobás and O. M. Riordan. "The Diameter of a ScaleFree Random Graph." Combinatorica 24:1 (2004), 5-34.

[Bollobás et al. 01] B. Bollobás, O. M. Riordan, J. Spencer, and G. Tusnády. "The Degree Sequence of a Scale-Free Random Graph Process." Random Structures and Algorithms 18:3 (2001), 279-290. 
[Buckley and Osthus 04] P. G. Buckley and D. Osthus. "Popularity Based Random Graph Models Leading to a Scale-Free Degree Sequence." Discrete Math. (2004) $282,53-68$.

[Dorogovtsev et al. 00] S. N. Dorogovtsev, J. F. F. Mendes, and A. N. Samukhin. "Structure of Growing Networks with Preferential Linking." Phys. Rev. Lett. 85 (2000), 4633.

[Drinea et al. 01] E. Drinea, M. Enachescu, and M. Mitzenmacher. "Variations on Random Graph Models for the Web." Technical report, Harvard University, Department of Computer Science, 2001.

[Erdős and Rényi 59] P. Erdős, A. Rényi. "On Random Graphs. I." Publ. Math. Debrecen 6 (1959), 290-297.

[Erdős and Rényi 60] P. Erdős and A. Rényi. "On the Evolution of Random Graphs." Magyar Tud. Akad. Mat. Kutato Int. Kozl. 5 (1960), 17-61.

[Gilbert 59] E. N. Gilbert. "Random Graphs". Ann. Math. Statist. 30 (1959), 1141-1144.

[Grechnikov 13] E. A. Grechnikov. "The Degree Distribution and the Number of Edges between Nodes of Given Degrees in the Buckley-Osthus Model of a Random Web Graph." Internet Mathematics 8:3 (2012), 257-287.

[Janson et al. 00] S. Janson, T. Łuczak, and A. Ruciński. Random Graphs. Wiley, 2000.

[Ostroumova and Grechnikov 12] L. Ostroumova and E. Grechnikov. "The Distribution of Second Degrees in the Bollobás-Riordan Random Graph Model." Moscow J. Combin. Number Theory 2:2 (2012), 85-110.

[Page et al. 98] L. Page, S. Brin, R. Motwani, and T. Winograd, "The PageRank Citation Ranking: Bringing Order to the Web." Technical report, Stanford University Database Group, 1998.

[Rudas et al. 07] A. Rudas, B. Tóth, and B. Valko. "Random Trees and General Branching Processes." Random Structures and Algorithms 31 (2007), 186-202.

[Talagrand 96] M. Talagrand. "Concentration of Measures and Isoperimetric Inequalities in Product Spaces." Publications Mathématiques de l'I.H.E.S. 81 (1996), 73-205.

[Zhukovskiy et al. 12] M. Zhukovskiy, D. Vinogradov, Y. Pritykin, L. Ostroumova, E. Grechnikov, G. Gusev, P. Serdyukov, and A. Raigorodskii. "Empirical Validation of the Buckley-Osthus Model for the Web Host Graph: Degree and Edge Distributions." Proceedings of the 21st CIKM (2012), 1577-1581.

Andrey Kupavskii, Department of Discrete Mathematics, Moscow Institute of Physics and Technology, 9 Institutskiy per., Dolgoprudny, Moscow Region, 141700, Russia (kupavskii@yandex.ru)

Liudmila Ostroumova, Yandex, Department of Theoretical and Practical Research, 16, Leo Tolstoy St., Moscow 119021, Russia (ostroumova-la@yandex.ru)

Dmitriy Shabanov, Moscow State University, Faculty of Mechanics and Mathematics, Department of Probability Theory, GSP-1, Leninskie gory 1, Moscow, 119991, Russia (shabanov@mech.math.msu.su)

Prasad Tetali, School of Mathematics and School of Computer Science, Georgia Institute of Technology, North Avenue, Atlanta, GA 30332, USA (tetali@math.gatech.edu) 